\title{
Towards a Cognitive and Neurobiological Model of Motivated Forgetting
}

\author{
Michael C. Anderson and Ean Huddleston
}

\begin{abstract}
Historically, research on forgetting has been dominated by the assumption that forgetting is passive, reflecting decay, interference, and changes in context. This emphasis arises from the pervasive assumption that forgetting is a negative outcome. Here, we present a functional view of forgetting in which the fate of experience in memory is determined as much by motivational forces that dictate the focus of attention as it is by passive factors. A central tool of motivated forgetting is retrieval suppression, a process whereby people shut down episodic retrieval to control awareness. We review behavioral, neurobiological, and clinical research and show that retrieval suppression leads us to forget suppressed experiences. We discuss key questions necessary to address to develop this model, relationships to other forgetting phenomena, and the implications of this research for understanding recovered memories. This work provides a foundation for understanding how motivational forces influence what we remember of life experience.
\end{abstract}

Keywords Recovered memories • Retrieval-suppression • Motivated forgetting - Neuroimaging and memory control

Over the last century, experimental research on memory has focused on passive factors that make us forget. Emphasis has been given to hypotheses about simple changes that happen to people such as the passive decay of memory traces, the accumulation of similar interfering experiences in memory, and changes in environmental context. This emphasis fits most people's view forgetting as undesirable, and that anything that increases the chances of it occurring surely must not be purposeful. In contrast, one fundamental issue of this volume is whether some of the forgetting that human beings experience may not be accidental, but rather may be produced by the

M.C. Anderson $(\varangle) \bullet$ E. Huddleston

MRC Cognition and Brain Sciences Unit, University of Cambridge, England, UK

e-mail: michael.anderson@mrc-cbu.cam.ac.uk 
desire to forget unpleasant events in life. More specifically, this volume is concerned with the forgetting and later recovery of memories of childhood abuse, and with explaining the nature of these experiences. Do such experiences reflect motivated forgetting? If so, how might this have been accomplished? These are some of the key questions that drive the recovered memory debate. In considering these questions, and the broader issue of motivation and memory, we present data relevant to a functional view of forgetting that diverges with the historical emphasis on passivity.

There can, of course, be little doubt that a motive to forget exists in all of us. People usually do not reminisce about unpleasant events, such as embarrassing incidents, quarrels, or physical discomfort. Some memories we would simply prefer to forget. Indeed, dwelling on major setbacks such as the death of a loved one, accidents, or significant personal failures can precipitate depression or anxiety. Such experiences are uninvited tenants in our memories, intruding into awareness when least expected, awakening our need to self-regulate. We are all are familiar with this process; an unwelcome reminder evokes a brief flash of experience and feeling, abruptly followed by efforts to evict the intruding memory from awareness and redirect our attention towards more pleasant thoughts. We do this to preserve our emotional state, to enhance our well-being, and to protect our sense of self; and sometimes, we do this simply to concentrate on what needs to be done in the present moment. These observations are so basic and universal as to be beyond dispute.

What people can disagree about, however, is whether limiting awareness of unwanted memories makes us forget them. On the one hand, people would be unhappy if they didn't have a way of forgetting the day-to-day unpleasantness of life. On this level motivated forgetting is obvious and adaptive. On the other hand, intuitions diverge about whether unusual and disturbing experiences can be forgotten. It is difficult for the average person to imagine how something like childhood sexual abuse could be forgotten; our instinct is "if that happened to me, I'd remember it." One is tempted to dismiss such reports as false, or perhaps not so much forgetting as denial of what one has always remembered. Yet, therapists claim to routinely observe forgetting on this scale. To many of them, motivated forgetting is an obvious fact of mental life. It is easier for them to believe that such forgetting is possible, because their experiences with patients are vivid and close to real circumstances; but the value of such cases has been challenged as scientific evidence, and this lies at the heart of the recovered memory debate. Are memories recovered in therapy genuine, or do they reflect suggestions by therapists, inclined to explain symptoms in terms of abuse? Clearly, progress on assessing the reality of recovered memories cannot rest solely on intuition or clinical observation.

In this article, we will consider how such experiences may emerge from motivational forces that shape retention, via mechanisms of cognitive control. In particular, we consider whether people's tendency to limit awareness of unwanted memories might cause forgetting, and whether laboratory science on this question can address the status of recovered memories. The ability to study motivated forgetting in the laboratory might seem limited by the assumption that it engages processes uniquely tied to trauma, and the consequent ethical difficulties of inducing trauma in controlled studies. In our research, we reject this tethering; instead, we assume that the 
processes underlying motivated forgetting are manifestations of broad cognitive control processes widely acknowledged to be crucial in the direction of action and thought. This assumption permits the decoupling of cognitive processes involved in motivated forgetting from trauma, enabling controlled study. We review the laboratory evidence on memory control via retrieval suppression, with an eye towards considering the mechanisms that might underlie some cases of recovered memories. We argue that although no linkage between retrieval suppression and recovered memories has been established (or sought), the mechanisms described here could potentially produce such experiences, under the right circumstances. We encourage further careful investigation of this issue.

\section{An Observation and a Hypothesis}

The current work originated from an invitation to discuss research on memory inhibition in relation to cases in which people reported recovering, often through therapy, long-forgotten memories of childhood sexual abuse. In the 1990s, such cases generated controversy, and a debate ensued in psychology about their origins and legitimacy. Early in this recovered memory debate, experimental psychologists primarily raised reasons to doubt the reality of the supposed memories underlying these recovered memory reports. This emphasis stemmed from a vivid appreciation of how fallible memory can be, and a reasonable suspicion about the dangers of overly suggestive therapeutic practices that might lead people to believe they had experienced something when they hadn't. Building on a strong body of research on suggestibility, experimental psychologists supported a skeptical stance to reports of recovered memories.

Though skepticism was clearly warranted, experimental psychology's response to this debate was initially one sided. The harm that suggestive therapy may cause to patients and their families needed to be mitigated, but neglecting the possibility that recovered memories might, in some cases, be real seems to go too far. If some fraction of cases is real, there is a hazard to past and future victims that must also be addressed. To consider this alternative, a conference was held entitled Trauma and Cognitive Science, one of the aims of which was to encourage cognitive psychologists to consider mechanisms that could produce authentic recovery experiences. The first author was invited to discuss his work on inhibitory control in memory in support of that aim. Might there be a motivated forgetting process underlying recovered memories that builds on general inhibition mechanisms that are of broad use in cognitive control?

The idea behind this possibility is straightforward. In our mental lives, we often need to suppress activity of responses or thoughts that interfere with our goals. For example, we often need to select one particular response from amongst a set of competitors vying for control over behavior. One solution to the problem of response selection is an inhibitory control process that de-activates the interfering response, rendering it non-interfering (see Anderson \& Weaver, 2009 for a review). A similar 
inhibitory control process may be engaged during memory retrieval. Parallel issues of selection arise when we are recollecting personal experiences, and so selectively retrieving a desired memory may require inhibition of similar competing ones. For instance, recollecting where we parked today may require inhibition of similar parking events (where you parked yesterday) that interfere with retrieval. Consistent with this, the first author's earlier work on retrieval induced forgetting had shown that retrieving some items from memory impaired retention of related items (Anderson, Bjork, \& Bjork, 1994). The impaired recall of competing memories appears to be produced, in part, by inhibitory control mechanisms that suppress distracting traces. The persisting effect of inhibition on competing memories renders them less accessible on later memory tests. Retrieval induced forgetting, initially observed with simple verbal materials (Anderson et al., 1994) has been generalized to a range of verbal and non-verbal materials (see Anderson, 2003; Levy \& Anderson, 2002, for reviews), suggesting that inhibitory control may be an important general factor in producing unintended forgetting.

Although the inhibitory control hypothesis of forgetting was developed independently of the recovered memory debate, it is striking how much resemblance there is between the processes required to explain the laboratory data and the ones that could explain motivated forgetting. The inhibitory control hypothesis proposes a controllable process for suppressing distracting memories that renders them less accessible for a functional purpose. Although the initial framing of this functional purpose had focused on resolving interference during retrieval, inhibitory control might also be useful in suppressing memories that are unwanted because they are uncomfortable. Thus, simply broadening the scope of situations in which inhibitory control might be engaged led to a plausible mechanism for controlling unwanted memories. Indeed, this extension of inhibitory control naturally follows from the broader conceptualization of retrieval inhibition as an adaptive process (Bjork, 1989; see Benjamin, 2010 for reviews), and, in particular, from work on directed forgetting (Bjork, 1972; Bjork, Bjork, \& Anderson, 1998; Geiselman, Bjork, \& Fishman, 1983, Johnson, 1994; see Golding \& MacLeod, 1998, for a historical review; see also later section entitled "Integration with Research on Directed Forgetting"). Could a person motivated to forget capitalize on retrieval-induced forgetting, and does any pattern of data in the clinical literature fit such a mechanism?

In considering this question, we came across an intriguing and counter-intuitive finding reported in Jennifer Freyd's (1996) book, Betrayal Trauma Theory: The Logic of Forgetting Childhood Abuse. Freyd argues that amnesia for sexual abuse may often reflect adaptive responses of a child who has been abused by a trusted caregiver. A child abused by a relative often has few options about how to respond. It thus may be in the child's best interests to forget the abuse if remembering it disrupts their ability to maintain attachment relationships with the caregiver. If so, one might expect to see more amnesia for abuse perpetrated by family members than for abuse by strangers, for whom no attachment relationships exist. This pattern has been observed. In a re-analysis of several data sets (Cameron, 1993; FeldmanSummers, \& Pope, 1994; Williams, 1994), Freyd (1996) found greater rates of selfreported forgetting of childhood sexual abuse when the perpetrator was a family 
member. For instance, in Cameron's (1993) study, $72 \%$ of people abused by a parent reported a period of forgetting followed by recovery, whereas only $19 \%$ of those abused by a non-parent reported any period of forgetting. Similar patterns were observed in Feldman-Summers and Pope's data (53\% versus 30\% forgetting for those abused by a parent or stranger respectively). Increased subjective reports of forgetting for caregiver-related abuse has been subsequently reported in other studies (e.g., Freyd, DePrince, \& Zurbriggen, 2006; Schultz, Passmore, \& Yodor, 2003; see Freyd, DePrince, \& Gleaves, 2007; DePrince et al., 2012, this volume, for reviews), although it has not been in observed samples in which abuse was likely to have been publicly disclosed in childhood (Goodman et al., 2003).

Taken at face value, these data are nothing less than astonishing. To appreciate why, one only needs to consider asking the average person who would be more likely to forget a particular past experience: someone who lived with reminders to that experience on a daily basis, or someone who was able to escape reminders for many years. Nearly everyone would say that the person who lived with reminders would have exceptional memory for the event, unavoidably so, because of the constant reminding. Yet, people abused by a family member are in precisely this situation - they must live with the abuser for years. Despite being in the presence of constant reminders of abuse, people abused by a family member are far more likely to report having had a period of forgetting, followed by recovery. Of course, people's retrospective claims about whether they forgot these memories might be doubted, and it is prudent to take these self-reports with a measure of skepticism (see, e.g., McNally, 2007 for arguments). Nevertheless, if this pattern truly reflects people's memory, it cries out for explanation, because it would seem to defy common sense and the established benefits of reminders. How could this be?

Upon reflection, we realized that understanding this counter-intuitive finding may lie in motivation. It seems uncontroversial to assume that the victim would be motivated to keep the abuse out of mind, regardless of who the perpetrator is. Importantly, however, the person abused at the hands of a family member faces a far greater and more consistent challenge in achieving this goal precisely because reminders to it would be inescapable, perhaps for many years. Keeping the abuse out of mind despite constantly confronting reminders requires a way to stop the reminder from eliciting the trace, and a way to retrain memory to elicit other thoughts upon seeing the abuser. Basically, if one cannot escape reminders, one must adapt one's internal landscape. We proposed that this was accomplished by retrieving diversionary thoughts unrelated to the abuse when the abuser is present, which we called the selective retrieval hypothesis (Anderson, 2001; see also, Bjork et al., 1998). If Freyd is correct, this motivated selective retrieval of non-abuse information would be especially likely in the case of parental abuse. The child would have powerful motives for not thinking of the abuse: if they are to sustain a necessary attachment relationship with the parent, the abuse cannot be on their minds, as it would undermine the ability to behave and feel appropriately. Thus, when motives to control awareness are present, constant reminders actually set the occasion for the engagement of processes that limit awareness of the memory, impairing retention. 
Identifying inescapable reminders as a key trigger for inhibition raised the more general issue of how and whether people can stop the retrieval process at all. Perhaps retrieving alternative memories was only one way of engaging an inhibition process whose primary function was to shut down retrieval. By this view, one might be able, when confronted with a reminder, to stop or suppress retrieval directly without retrieving diversionary thoughts. Virtually no research had been done on this issue. So, motivated by this intriguing pattern we decided to study retrieval stopping as the elemental process that may underlie memory control, and that may be the foundational response to confronting inescapable retrieval cues. This led us to focus on developing a functional model of motivated forgetting based on controlled experiments on retrieval stopping.

\section{A Functional Model of Motivated Forgetting}

After an unpleasant experience, unwanted memories of the event tend to intrude into awareness. Indeed intrusive memories seem to leap to mind in response to reminders, despite attempts to avoid those memories. This reminding has a reflexive quality similar to habitual actions, and like habitual actions, we often try to stop them. Consider an example of motor stopping. One evening, the first author accidentally knocked a potted plant off his window sill. As his hand darted to catch it, he realized that the plant was a cactus. Mere centimeters from it, he stopped himself from catching the cactus. The plant fell and was ruined, but he was relieved to not be pierced with little needles. This example illustrates the clear need to have the ability to override a strong reflexive response, which is a basic function of cognitive control. Without the capacity to override prepotent responses, we could not adapt behavior to changes in our goals or circumstances. We would be slaves to habit and reflex.

Like automatic actions, people often attempt to stop the retrieval process. In the framework that guides the current work, we have proposed that this functional similarity between memory and motor stopping is important and provides a theoretical basis for understanding memory control. Under this view, retrieval and motor stopping constitute special cases of the broader ability to override prepotent responses, and the mechanisms underlying the two are similar, if not the same (Fig. 1). Because retrieval stopping is an elemental process underlying motivated forgetting, this view

Fig. 1 (continued) instead be made (e.g., S-R compatibility and antisaccade tasks). As shown in (d), if no alternative response is warranted, the movement can simply be canceled (e.g., go/no-go, stop-signal, and countermanding saccade tasks). As shown in (e), sometimes inhibition must be initiated to selectively retrieve a memory with a weaker association to a cue that is shared by another trace (e.g., retrieving today's parking spot and suffering interference from the memory of yesterday's; RIF). In other circumstances (f), confronting a cue may activate an unwanted memory, leading the person to stop retrieval. For instance, when the sight of a picture of a person initiates retrieval of an unpleasant memory, retrieval might be stopped. This process can be assayed by TNT tasks. RIF, retrieval-induced forgetting; S-R, stimulus- response; TNT, think/no-think (Reprinted with permission from Anderson \& Weaver, 2009, copyright (C) 2009 Elsevier Ltd.) 


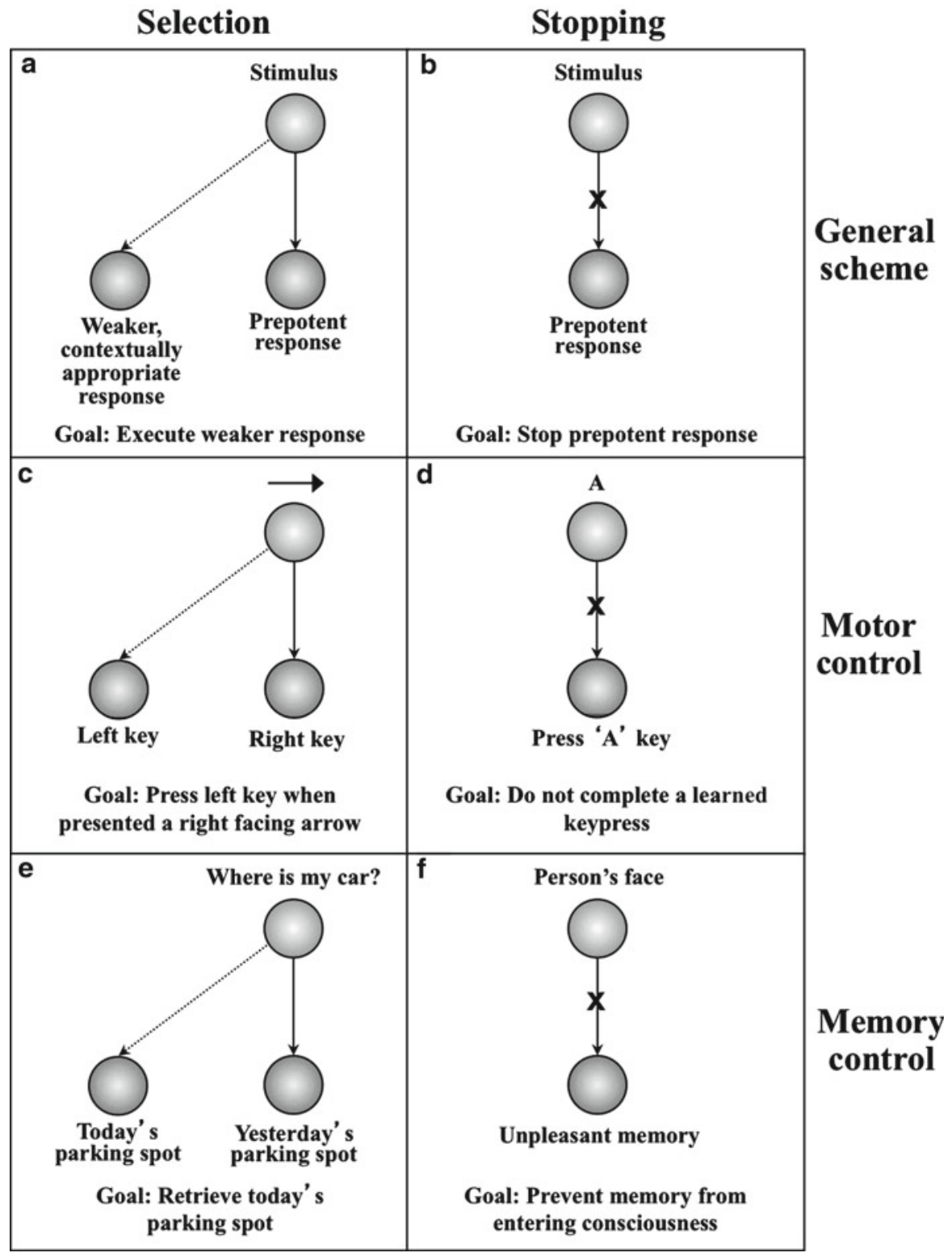

Fig. 1 Two situations that require response override in human action and thought (selection and stopping) and commonly used paradigms. The top row represents a schematization of these two situations. In each instance, a stimulus is associated with one or more responses, such that when the stimulus appears, the responses become active in proportion to their associative connection to the cue (represented by thickness of the line). In selection (a), the weaker response must be made, despite the existence of a strong competitor that becomes more active and threatens to capture control of behavior. In stopping (b), there is only one response, but it must be prevented. As shown in (c), sometimes the prepotent action is not the correct response, and a nondominant movement must 
situates theorizing about this phenomenon squarely in the realm of cognitive control, one of the most widely studied areas in cognitive neuroscience. Indeed, one of most broadly accepted functions of cognitive control is to override automatic responses to stimuli when they are inappropriate (Luria, 1966; MacDonald, Cohen, Stenger, \& Carter, 2000; Norman \& Shallice, 1986). Within this framework then, research on retrieval stopping can be informed by cognitive and neurobiological research on how humans and non-human primates override reflexive, prepotent actions.

But how do humans and other organisms keep from being controlled by habitual actions? One widely discussed possibility is that we inhibit undesired actions to stop them. The function of this hypothetical inhibition process is much like the role of inhibition in response selection discussed previously, serving to limit activation of an undesired response. By this view, when we encounter a stimulus, "activation" spreads from that cue to possible responses. Activation can be thought of as the amount of "energy" a response has, influencing its accessibility; a response will be emitted once it is sufficiently activated. If one wishes to override the response, one may engage inhibitory control, a subtractive mechanism that reduces the response's activation. If motor actions are stopped in this manner, perhaps we control unwanted memories in a similar way. Like actions, memories can be triggered by activation spreading from reminders that we encounter. Might inhibition be recruited to stop retrieval, allowing us to avoid catching our "mental cacti"? If so, how would we study this question?

\section{Stopping Retrieval: Basic Behavioral Findings}

To study how people stop retrieval, Anderson and Green (2001) developed a procedure modeled after the widely used go/no-go task, a paradigm designed to investigate motor stopping. In a typical go/no-go task, people press a button as quickly as possible whenever they see a letter appear on a computer screen, except when the letter is an X, for which they are to withhold their response. Their ability to withhold the response measures inhibitory control over action (e.g., how well a person avoids catching the cactus). To see whether stopping retrieval also engages inhibitory control, Anderson and Green (2001) adapted this task to create an analogous procedure for studying memory control called the think/no-think paradigm.

The situation faced by participants in the think/no-think paradigm mimics situations in which we stumble upon a reminder to a memory that we prefer not to think about, and try to keep it out of mind. Participants study cue-target pairs (e.g., ordeal - roach), and are trained to recall the second word (roach) whenever they encounter the first word as a reminder (ordeal). Participants are then asked to exert control over retrieval during the think/no-think phase (Fig. 2). Most trials require them to recall the response whenever they see the reminder (hereinafter referred to as "Respond Trials" or sometimes "Think Trials"), but for certain reminders, participants are admonished to avoid retrieving the response (hereinafter referred to as "Suppress Trials" or sometimes, "No-Think Trials"). It is emphasized that it is 
Think/No-Think Paradigm

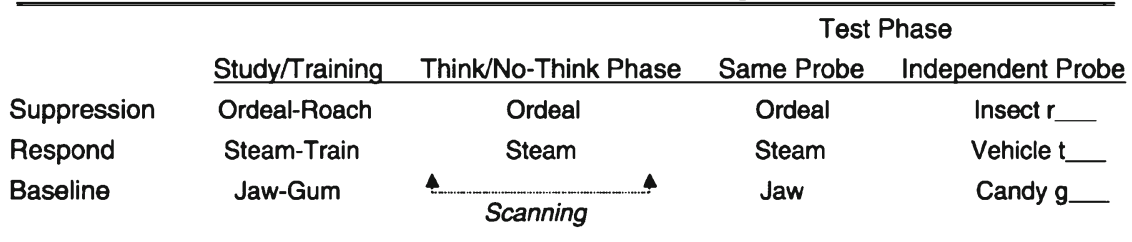

Fig. 2 Depiction of the think/no-think procedure. In the training phase participants study numerous word pairs, so that when they are presented with the left hand word they are able to recall the right hand word. Next, in the Think/No-Think (TNT) phase, for some left hand words (Ordeal), participants' task is to recall and think about the right hand word. However, for other left hand words (Steam), participants' task is to prevent the right hand word from coming to mind at all. A final group of word pairs act as baseline pairs, with no reminders being presented during the TNT phase. During the final test phase, participants' memory for the right hand words is tested in two ways. In the Same Probe test, the original left hand word is presented, and participants must recall the associated right hand word. In the Independent Probe test, a novel category cue is presented along with a letter stem, and participants must recall the studied word that is a member of that category that begins with the designated letter. (From Anderson et al., 2004, reprinted with permission from AAAS)

insufficient to avoid saying the response - they must prevent the memory from entering awareness altogether. Thus, to achieve this task, participants have to stop the cognitive act of retrieval. Can people recruit inhibitory control to prevent the memory from intruding into consciousness?

Since awareness cannot be observed, it is difficult to know whether a person truly prevents a memory from entering consciousness. Instead, the think/no-think procedure measures the aftereffects of stopping retrieval, based on the idea that inhibition of the unwanted memory might linger, making these memories harder to recall. To assess this behavioral footprint of suppression, a final test is given in which participants again see each reminder and are asked to recall every response they learned earlier. The percentage of originally studied items that are correctly recalled on this final test is computed separately for each condition. If stopping retrieval engages inhibitory control processes, we should find poorer recall of Suppress items on a later test. If so, it would suggest that people's common tendency to suppress awareness of unwanted memories in response to reminders may in fact have measurable aftereffects on the later retention of the suppressed trace, consistent with the existence of a motivated forgetting process.

Research using the Think/No-Think procedure documents a number of central facts about the effects of suppressing retrieval. Figure 3 illustrates these keys facts. Figure 3 (left) reports the results of a combined analysis of studies conducted in our own laboratory, irrespective of whether they were published or unpublished, and was first reported in Anderson and Levy (2006). Figure 3 (right) illustrates all data published to date, irrespective of laboratory, aggregating over 47 experiments from 32 articles (see Appendix A for listing), with 1669 participants measured in on the Same Probe test and 800 participants measured on the Independent Probe test (to be described in next section). These analyses are restricted to neurologically 

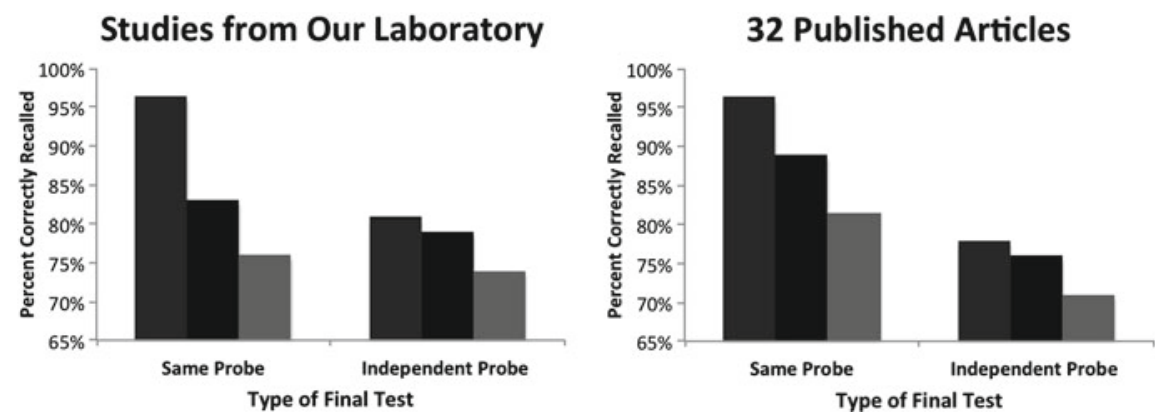

Fig. 3 Left panel: a meta-analysis of published and unpublished TNT studies run in our laboratory over multiple years. Right panel: data from 32 published articles for which full TNT data was reported on recall tests. For both panels, data are shown for the respond, baseline, and suppress conditions (in that order) for both the same probe and independent probe tests, when available. Only 180 participants overlap between the two analyses, with a total of 2,174 participants given the Same Probe test, and 1,305 participants given the Independent Probe test across the panels. Both data sets are restricted to neurologically and psychiatrically normal young adults. Data from the "Respond" and "Suppress" conditions were taken from the highest level of repetition used in a given study (most studies used 12 and 16 as maximum repetition values for Respond or Suppress trials). Four additional studies were not included in the right panel because (a) they lacked any behavioral data and focused only on imaging (Butler \& James, 2010), (b) used an indirect memory test (Kim \& Yi, 2008), or (c) did not report data from all relevant conditions (Depue et al., 2006; Marx et al., 2009). For the 32 included studies, a weighted average across experiments was constructed for each condition, depending on sample size. Appendix A contains a full listing of all studies, with sample size, and all populations studied, including other specialized samples (e.g., depressed patients, ADHD) not included in the figure

and psychiatrically normal young adult participants and represent (combined over left and right panels) the data from 2,174 participants from one dozen countries (only 180 participants overlap between the two panels). This extremely large sample conveys several broad generalizations about the aftereffects of suppressing retrieval on unwanted memories. First, after retrieval suppression, "Suppress" items are recalled significantly less often than are "Respond" items (Fig. 3). This large difference ( $22 \%$ vs $15 \%$ in the two panels), known as the total control effect, demonstrates vividly how one's disposition towards reminders of an experience may modulate its later retention. When one is favorably disposed towards a memory, a reminder may trigger retrieval that enhances later retention. In contrast, when one is motivated to exclude a memory from awareness, the normal benefits of retrieval are dramatically reduced, indicating a high level of control over the effects of reminders on memory.

Although the total control effect demonstrates the intentional control of memory, it does not address how it is produced. For example, one cannot tell whether the total control effect reflects the benefits of positive attention to the retrieved trace, the detrimental effects of suppressing the unwanted memory, or both. It is possible, for example, that stopping retrieval does no harm to a memory, but merely stops the retrieval process from unfolding, thereby preventing the benefits of reminders on memory. 
Such a dynamic would still constitute an interesting an important determinant of which traces ultimately survive in memory because rehearsal and reactivation are key factors thought to enhance longevity of our experiences (Allen, Mahler, \& Estes, 1969; Bjork, 1975; Carrier \& Pashler, 1992; Karpicke \& Roediger, 2008; Landauer \& Bjork, 1978). Indeed, some have built the case that selective prevention of retrieval, by itself, is a key process of motivated forgetting (Erdelyi, 1996). Nevertheless, it is of interest to determine the separate positive and negative components to the effect, and, particularly, whether retrieval suppression has detrimental effects on the retention of unwanted memories.

To address these issues, the Think/No-Think paradigm includes a third set of pairs that are also studied initially, but that do not appear during the think/no-think phase. These pairs provide an estimate of how well participants would recall pairs given that they have neither retrieved nor suppressed memory for them in the intervening Think/No-Think phase and because they are studied and tested at the same time as Respond and Suppress pairs, they control for forgetting due to the passage of time. They thus provide a baseline condition (hereinafter referred to as "Baseline Items") for measuring both potential positive control effects, and negative control effects. A positive control effect would reflect enhanced memory for "Respond" items above that of Baseline items, and would confirm the expectation that reminders enhance later retention when people are inclined to remember. A negative control effect would reflect impaired memory for "Suppress" items below that of Baseline items arising from people's effort to stop retrieval. As Fig. 3 illustrates, both positive and negative control effects contribute to the total control effect. When considering the Same Probe data (i.e., when participants are cued on the final test with the same cue used to study the item), the average negative control effect is around $8 \%$ (range from $7 \%$ facilitation to $26 \%$ impairment across experiments), and the average positive control effect of 9-14\%. These two analyses make an extremely clear and consistent point: when people are motivated to avoid being reminded of an unwanted memory, reminders do not merely fail to enhance memory, they actually trigger processes that impair retention of the suppressed memory.

The negative control effect is striking and counterintuitive, particularly when one considers that reminders to the suppressed items are directly confronted by subjects up to 16 times per item during the Think/No-Think phase (compared to Baseline items, which receive no reminders). Thus, the negative control effect turns our expectation about the effect of reminders on its head and powerfully illustrates the effects of motivation on memory. Importantly, the negative control effect occurs even when people are paid a reward for each item they remember, making it extremely unlikely that people are simply withholding responses on the final test. The negative control effect is even observed when people are led falsely to believe (just prior to the final memory test) that we, as experimenters, hope to see improved memory for suppressed items, showing that the effect does not reflect subjects withholding items simply to conform to perceived expectations (Anderson \& Green, 2001). In contrast, asking people to merely avoid saying the response, instead of avoiding thinking about it, eliminates the negative control effect, isolating control over consciousness as the critical factor causing forgetting (Anderson \& Green, 2001). 
These findings establish a clear laboratory model through which one can study retrieval suppression. Understanding the mechanisms underlying retrieval suppression through this model task allows us to develop a theory of a core process involved in motivated forgetting, integrating this otherwise controversial process with fundamental and widely accepted mechanisms for controlling behavior. This theoretical framework may help us to understand when this type of forgetting will occur in clinical settings. Next we consider how this model task has been used to document core characteristics of the negative control effect that speak to the mechanisms that underlie it.

\section{Characteristics of the Negative Control Effect}

Although the negative control effect reveals a surprising level of control over memory retrieval, it could be produced in a number of ways. Since originally reported, however, a great deal has been learned about the characteristics of negative control effects, and what causes them, and also population differences in memory control. Here we discuss those characteristics and individual differences. Collectively, these findings support the view that the memory deficit is produced in part by an inhibitory control process acting on the unwanted memory, degrading its later retention. However, other processes are also likely to contribute, depending on how people approach the task of controlling awareness.

\section{Cue-Independence}

One characteristic that favors a role of inhibitory control in producing the negative control effect is the tendency for the forgetting to generalize to novel test cues. So, for example, if a participant had studied a pair such as "Ordeal-Roach," and then had suppressed "Roach" whenever they were cued with "Ordeal," later recall of "Roach" is impaired both when it is tested with Ordeal (i.e., Same Probe test), and a novel test cue such as Insect $\mathrm{R}$ - (i.e., Independent Probe test). This property, known as cue-independence, previously demonstrated in the context of retrievalinduced forgetting (Anderson \& Spellman, 1995, see Anderson, 2003 for a review), has been observed in a number of studies of retrieval suppression (Anderson \& Green, 2001; Anderson et al., 2004; Anderson, Reinholz, Kuhl, \& Mayr, 2011; Bergström, de Fockert, \& Richardson-Klavehn, 2009; Lambert, Good, \& Kirk, 2010; Murray, Muscatel, \& Kensinger, 2011; Paz-Alonso, Ghetti, Matlen, Anderson, \& Bunge, 2009; Tomlinson, Huber, Rieth, \& Davelaar, 2009; Tramoni et al., 2009). Figure $3 \mathrm{a}, \mathrm{b}$ document the general pattern observed on independent probe tests within our lab $(\mathrm{N}=687)$, and averaged across 800 participants in all published studies. The negative control effect for independent probes occurs in both these data sets, despite the fact that the cues provided are unrelated to those used to suppress the response initially. The median independent probe effect across these 1,305 


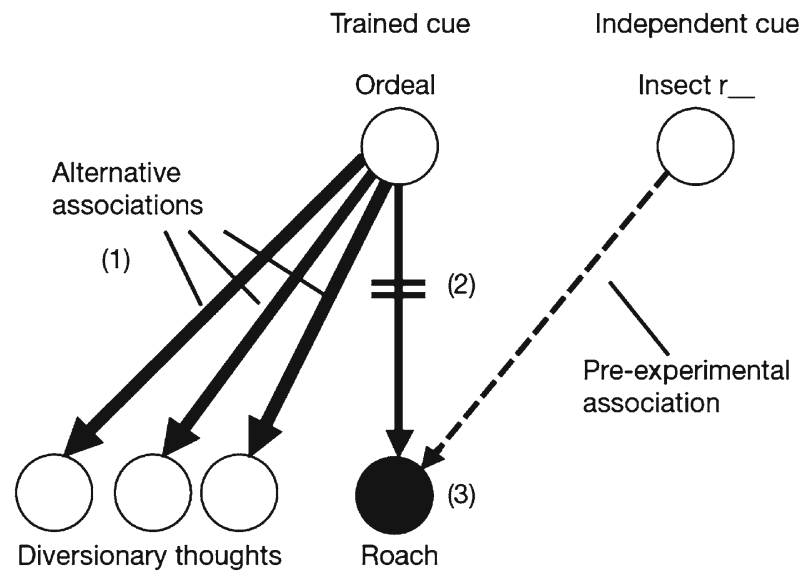

Fig. 4 Three mechanisms that can explain impaired recall in the same-probe condition, illustrated with a stimulus pair. Associative interference posits that suppression training leads subjects to generate diversionary thoughts (1) to the trained cue that interfere during later attempts to recall the target. Unlearning assumes that suppression training weakens the cue-target connection (2). The suppression hypothesis states that suppression training inhibits the target (3). Note that testing the target with an independent cue circumvents interference (1) and unlearning (2). Any impairment found with this test is likely to be localized to the target, consistent with inhibition (Reprinted with permission from Anderson \& Green, 2001, copyright (C) 2001 Macmillan Publishers Ltd.)

participants (across both panels) is about 6\%, slightly smaller than the typical effect observed for the Same Probe test (8\%). The total control effect, by contrast is noticeably smaller on independent probe tests, primarily due to the fact that positive control effects largely disappear on such tests, suggesting that facilitation of retrieved items is largely cue-dependent.

Cue-independence is a theoretically important feature of the negative control effect because it suggests that retrieval suppression alters the accessibility of the unwanted memory in a general way, consistent with inhibition. If an inhibitory control mechanism had truly suppressed the unwanted memory, reduced activation of the excluded trace may produce aftereffects irrespective of whether that trace was tested with the same cue used to induce suppression or a different one, as we observed. This pattern suggests that other accounts of the negative control effect in terms of associative interference are not sufficient. For example, one might have imagined that participants, in response to the reminder "Ordeal," might have generated alternative, diversionary thoughts in response to it to distract themselves (Fig. 4). If so, perhaps they have difficulty recalling "Roach" because "Ordeal" now instead reminds them of their distracting thoughts - a form of interference. Although this process may contribute to the effect when measured with the original cue (Ordeal), it seems unlikely to contribute on tests using a novel cue like Insect $R$ The fact that impairment generalizes to such cues suggests that inhibition contributes to the negative control effect (see, however, Tomlinson et al., 2009, for alternative view). 
Although the cue-independence property has been replicated many times, there have also been clear cut cases in which this effect has not be found, even when the negative control effect is found with the original cue (Ordeal). This suggests that the negative control effect on the Same Probe test may be driven by several mechanisms, some of which are non-inhibitory in nature. Although it is not yet clear what factors dictate when the effect will be inhibitory, one likely contributor appears to be the strategy that people adopt to control their memories, a topic to which we will return shortly. But the clear existence of cue independence in the general case over an exceptionally large sample (Fig. 3) suggests that a control mechanism exists that renders an unwanted memory less accessible through its inhibition.

\section{Thought Substitutes Increase the Effect}

In most studies using the Think/No-Think paradigm, participants receive no instructions as to how they should prevent retrieval of the unwanted memory. When we developed the procedure, we did not wish to presuppose that one strategy might be better than others, and wanted to allow participants to develop their own natural solutions to memory control. When no instructions are given, however the approach to the task can vary. Indeed, in a recent article (Levy \& Anderson, 2008), we documented many solutions (and their frequency in a large sample) that people use to avoid the unwanted memory, including perceptual analysis or phonological rehearsal of the cue, "mind blanking" and, of course, the generation of distracting words, thoughts, and memories related to the cue. In general, we have not observed correlations of these strategies with the negative control effect.

Other investigators, however, have argued that thought substitution is a superior method for forgetting unwanted memories, and have experimentally manipulated this behavior. In an early study, Hertel and Calcaterra (2005) gave participants alternative words to associate to the Suppress cues, and asked them to retrieve these "thought substitutes" as a way of preventing the unwanted memory from coming to mind whenever it's respective Suppress cue word appeared. They found a significantly larger negative control effect with thought substitutes (15\%) compared to an Unaided group, who received conventional Suppress instructions without substitutes $(0 \%)$, though the latter group was contaminated with non-compliant subjects who didn't obey the Suppress instructions (Hertel \& Calcaterra, 2005). Moreover, in the Unaided group, the negative control effect was significantly larger for participants who reported distracting themselves with alternative thoughts (12\%) compared to participants who reported not doing this (12\% facilitation) (see also Hotta \& Kawaguchi, 2009). Hertel and colleagues have reported robust negative control effects with thought substitutes (Hertel \& McDaniel, 2010; Joormann, Hertel, Lemoult, \& Gotlib, 2009; LeMoult, Hertel, \& Joorman, 2010). Although thought substitutes do not always produce larger negative control effects than in the unaided group (Hertel \& McDaniel, 2010), the tendency, in our combined analysis (Fig. 3, right) was for 
thought substitutes to produce larger effects on average $(13 \%, \mathrm{~N}=262)$ than are produced in an uninstructed condition $(7 \%, \mathrm{~N}=1407)$ on Same Probe tests. Indeed, thought substitution has, on some occasions produced impressively large effects (e.g., 30\%; Joormann et al., 2009).

Research on thought substitution demonstrates that learning to retrieve alternative diversionary thoughts in response to a reminder can be an effective way to hasten the forgetting of an unwanted memory. This finding fits well with the selective retrieval hypothesis (Anderson, 2001) of the enhanced forgetting of parental abuse described at the outset. According to that hypothesis, victims of abuse who are faced with inescapable reminders to an unwanted memory are forced into a situation of retraining their memory's response to the reminder, by selectively retrieving alternative thoughts and memories about the abuser. Hertel's work clearly models these conditions, inasmuch as the instruction to not think of the unwanted memory provides the motive, and the thought substitute, the target for selective retrieval. It remains to be seen whether thought substitution could be used to enhance forgetting of complex, realistic experiences.

One might take research on thought substitution as evidence that the negative control effect is caused exclusively by thought substitution, and, moreover, that this process may simply reflect associative interference. This possibility might seem to be supported by Hertel and Calcaterra's finding that only those Unaided subjects who reported using self-distraction as a strategy showed negative control effects. Although it is clear that thought substitutes can cause a negative control effect, a number of considerations indicate that these conclusions are premature. First, Hertel and colleagues' never studied the effects of strategies other than thought substitution, but rather focused on comparisons with an Unaided group. As such, we cannot tell whether the advantage of thought substitution in their studies and in our metaanalysis reflects something special about this strategy that enhances forgetting, or, instead, whether encouraging the consistent use of any strategy improves the effect. It seems likely that participants in the Unaided group took some time to refine their strategy over blocks in the TNT phase, and this variability may contribute to smaller effects. Second, the larger negative control effects for Hertel's Unaided subjects who used self-distraction are substantially driven by the non-compliant subjects in their study (i.e., subjects who deliberately did not follow Suppress instructions; see later section entitled The Negative Control Effect Sometimes Does Not Occur for a discussion), who obviously would not have used self-distraction. Finally, even if thought substitution induces a negative control effect, this by no means implies that the effect is driven by associative interference, but rather could reflect inhibitory processes associated with retrieval-induced forgetting. Indeed, prior work on retrieval-induced forgetting has established that the mere effort to retrieve a target, even if not successful, can induce inhibition of competing items, suggesting that one should not presume that thought substitution effects are driven by interference (Storm, Bjork, Bjork, \& Nestojko, 2006; see Storm, 2010 for a review). Evaluation of these possibilities would require the examination of strategies other than thought substitution. 


\section{Direct Suppression can Induce Negative Control Effects}

Although thought substitution instructions appear sufficient to induce negative control effects, it is unclear from Hertel's research whether they are necessary. In a particularly informative example of this point, Bergstrom et al. (2009) contrasted the effects of thought substitution and direct suppression on the negative control effect. In their thought substitution condition participants were asked to prevent retrieval of the Suppress items by generating their own thought substitutes in response to the cue words during the think/no-think phase. In the direct suppression condition, by contrast, participants were instructed NOT to distract themselves with thought substitutes, but rather to focus on the cue and actively block out the unwanted associate if it happened to come to mind. If thought substitution is necessary to produce negative control effects, one should find memory impairment only in the thought substitution group. If an inhibitory control process contributes to the suppression of unwanted memories, however, negative control effects might be observed in both groups.

The results of this study, illustrated in Fig. 5, are striking and informative. Participants who generated thought substitutes in response to the cue words showed significant negative control effects, as one might expect from prior research. More interestingly, however, the direct suppression group showed these effects as well, and to no less an extent than subjects using thought substitution. Moreover, unlike participants who were instructed to generate thought substitutes, subjects who engaged in direct suppression showed negative control effects that generalized to independent probe test cues. Thus, direct suppression yielded cue-independent forgetting, whereas thought substitution did not. To the extent that cue-independence can be taken as a marker for the inhibitory control, these data suggest that instructions to directly expel a memory from awareness are implemented by an inhibitory process that suppresses the unwanted trace.

One might wonder whether Bergstrom et al.'s findings are truly caused by a direct suppression process or, instead, might reflect the generation of thought substitutes that went unmonitored by the experimenters. Two aspects of their data argue against this interpretation. First, the direct suppression group showed a qualitatively distinct pattern of forgetting, with generalization to independent cues, not experienced by subjects who were directly instructed to generate thought substitutes. If uncontrolled thought substitution underpinned this effect, one would not expect this functional dissociation. Second, Bergstrom et al. showed that direct suppression and thought substitution were dissociable electrophysiologically. As will be discussed in more detail later, direct suppression, but not thought substitution modulated the parietal Episodic Memory effect, which a large body of research has established as a reliable marker of recollection (Friedman \& Johnson, 2000). Thus, direct suppression yielded little evidence of retrieval-related activity, whereas thought substitution did. Similar electrophysiological and behavioral effects of direct suppression have been found by others (Hanslmayr, Leipold, Pastötter, \& Bäuml, 2009; Hanslmayr, Leipold, \& Bauml, 2010).

Taken together, these findings indicate that direct suppression is sufficient to induce negative control effects, and may be accomplished in a qualitatively different 


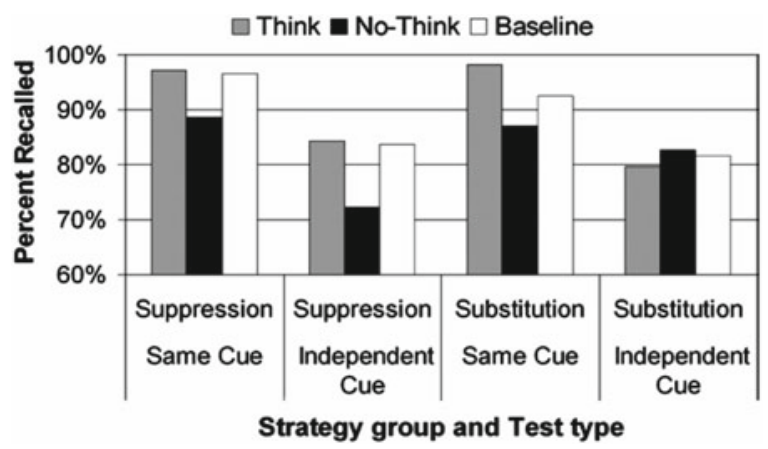

Fig. 5 Final recall data for the same-probe ("same-cue") and independent-probe ("independent-cue") tests, from Bergstrom et al., 2009. Both groups showed a significant Suppress impairment compared to baseline on the same-cue test, but only direct memory suppression impaired Suppress recall compared to baseline on the independent-cue test (Reprinted with permission, copyright (C) 2009 Elsevier)

way than thought substitution. Across the 96 participants ( 3 papers) in which this procedure has been used, the negative control effect averages around 9\% slightly less than the effect observed for thought substitution, but an improvement over providing no specific instructions. Given the clear evidence that direct suppression induces cue-independent forgetting, these data provide strong indication that inhibitory control processes are involved in helping to expel unwanted memories from awareness. Importantly, these processes do not require retrieval of thought substitutes to be engaged.

\section{Advance Warning Enlarges the Effect}

Several studies by Hanslmayr and colleagues have shown that negative control effects increase when participants are given advance warning that an upcoming trial will require them to suppress retrieval, at least with direct suppression instructions. For example, Hanslmayr et al. (2010) asked participants to learn face-word associations and then perform the Think/No-Think task. In the preparation group, each Respond and Suppress trial was preceded by a 1-second task cross that was either colored red (Suppress) or green (Respond) to warn participants of the nature of the upcoming trial. The no-preparation group received no advance warnings. Hanslmayr et. al. found a $17 \%$ negative control effect in the prepared group, compared to a $3 \%$ effect in the unprepared group. In a related study that will be discussed more later, Hanslmayr et al. (2009) found that when participants receive a warning cue about an upcoming Suppress trial, electrophysiological markers of episodic retrieval mode in right prefrontal cortex (Duzel et al., 1999) are significantly reduced in preparation for the upcoming trial, and that the extent of this reduction predicts later negative control effects on the final test. 
Effects of advance warning on memory suppression suggest that people can pre-engage the neural machinery necessary to directly suppress the retrieval process, thereby enhancing the efficacy of memory control. One can imagine how knowing in advance that one is likely to confront unwelcome reminders might help one to "steel" oneself against the unpleasant effects of those reminders. Thus, environments in which the appearance of these reminders is predictable and unavoidable might be expected to lead to larger negative control effects than environments where reminders are less predictable.

\section{Negative Control Effects Build with Repetition}

Many studies have found that the size of the negative control effect increases with the number of times people attempt to suppress retrieval. For instance, averaged over the three studies $(\mathrm{n}=96)$ in Anderson and Green (2001), participants recalled $87 \%, 85 \%, 83 \%$ and $80 \%$ of the items after $0,1,8$, and 16 suppression attempts. More recently, Anderson, Reinholz, Kuhl, and Mayr (2011) found 84\%, 81\%, 79\%, and $76 \%$ across the same levels of repetition for younger adults. Similar parametric functions have been found by others (Joormann et al., 2009; Kim, Yi, Yang, \& Lee, 2007; Hanslmayr, Leipold, \& Bauml, 2010 ; Joormann, Hertel, Brozovich, \& Gotlib, 2005; Lambert et al., 2010; Lee, Lee, \& Tsai, 2007). Similar patterns are found on the Same and the Independent Probe tests (Fig. 6), and improvements with practice have been observed with thought substitution, direct suppression and without any particular suppression instructions.

Although negative control effects generally build with repetition, the functions that relate repetitions to the size of the effect are not well characterized, and there also appears to be variability in the patterns. For example, some studies have observed very gradual build-ups of impairment with repetition such as the ones mentioned above; others have found sizeable negative control effects after just a few repetitions, with very modest increases in the effect after a much larger number (e.g., 21\% after 2 repetitions, 22\% after 12 for Depressed subjects in Joormann et al., 2009). And sometimes, even when there is a gradual build-up, there is noise in the function, with unexpected increases in recall with larger numbers of repetitions, followed by decreases (e.g., 83\%, 78\%, 84\%, and 74\% after 0, 1, 8, and 16 repetitions respectively, in Anderson et al. 2011). In general, however, when one considers a larger sample, the function increases monotonically with repetition for young healthy adults, suggesting increasing efficacy with repetition.

The reasons for these variations have not yet been established, but two possibilities seem likely. First, many of the studies that show large early effects followed by minimal increases in the effect appear to be ones in which thought substitutes are provided by experimenters before the TNT phase has begun. Perhaps the large effect after a few repetitions represents the contributions of having just studied the thought substitutes, and the tendency for them to be mistakenly provided on the final test when the original cue is given. This explanation fits with the fact that such unusual functions are usually observed on Same Probe tests and not Independent Probe tests, 

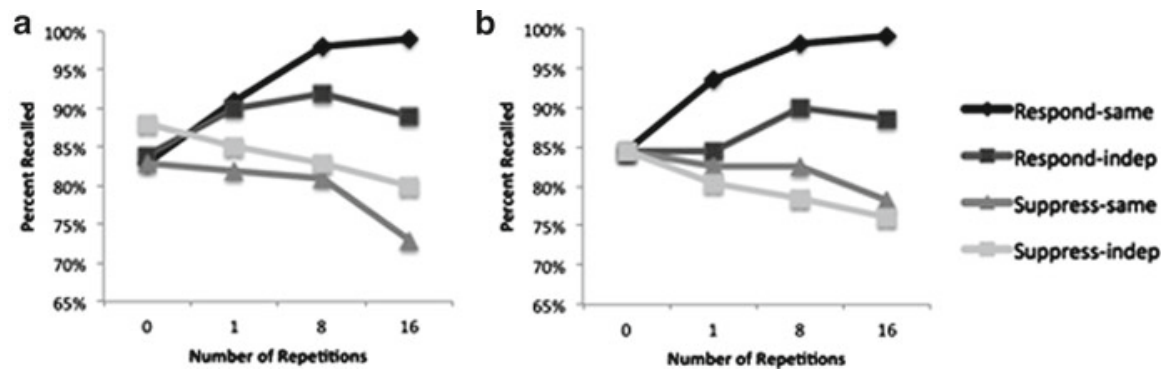

Fig. 6 Final recall for respond and suppression items as a function of the number of repetitions for the Same-probe and Independent-probe tests. (a), Anderson \& Green, 2001 (Adapted by permission, copyright (C) 2001 Macmillan Publishers Ltd.); (b), Anderson et al., 2011. Note that negative control effects increases monotonically with repetitions on both the Same Probe and Independent Probe tests

which are highly constrained in the answers they allow. A second source of variability may be the overall duration of the Think/No-Think phase itself, and the contributions of fatigue. Because suppressing retrieval requires cognitive control, and because the Think/No-Think phase itself can last 25-40 min, subjects' efforts at retrieval suppression surely wane as blocks progress. If participants lapse at suppression in later blocks, they may allow yet-to-be inhibited Suppress items to intrude, causing facilitation on those items. Conditions with larger number of repetitions may include more of these failures, yielding a noisier function.

These observations suggest that experimenters would be wise to keep subjects consistently motivated during the Think/No-Think task, and to provide short rest breaks throughout. This would seem especially important when comparing populations on their inhibitory control abilities, if these populations vary in motivation or vigilance. Clinically, it is interesting that memory control may be vulnerable to conditions that lead to distraction or fatigue, such as depression, circadian arousal, or sleep loss.

\section{Generalizes to Non-Verbal Materials}

Although the majority of published studies have used verbal pairs, many studies have demonstrated negative control effects with other materials. Studies have used face-word pairs (Depue, Banich, \& Curran, 2006; Hanslmayr et al., 2010; Hanslmayr et al., 2009), word-face and word-place pairs (Detre, Natarajan, \& Norman 2010; Huddleston \& Anderson, in preparation), word-line-drawing pairs (Kim \& Yi, 2008), and face-scene pairs (Depue et al., 2006; Depue, Curran, \& Banich, 2007; Depue, Banich, Burgess, Willcut, \& Ruzic, 2010). For example, in a study by Depue et al. (2007), participants studied pairs composed of faces and complex scenes varying widely in content, and were trained on these pairs until they could recognize the scene that went with each face. During the Think/No-Think phase, participants were 

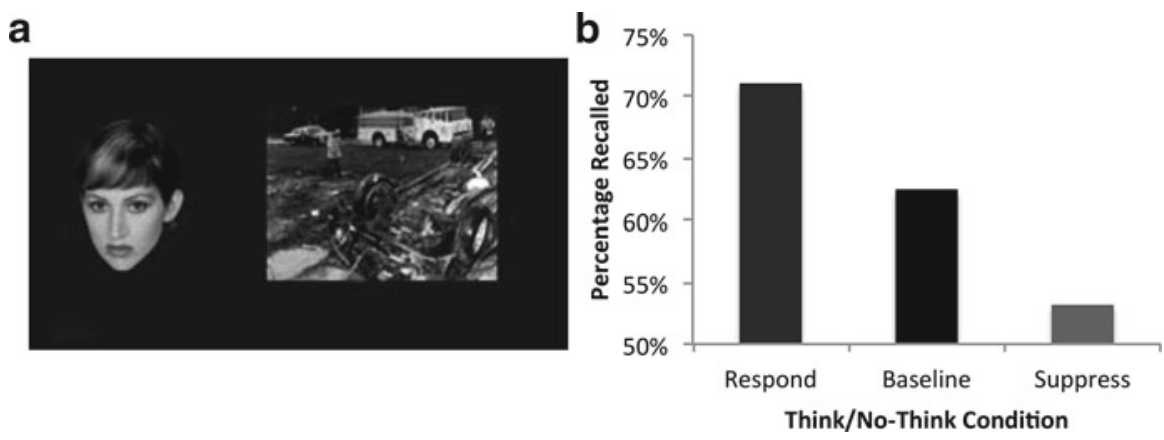

Fig. 7 (a), Depue et al., 2007 used faces for cues and both negative and neutrally valenced complex scenes as targets (reprinted with permission from AAAS); (b), final recall performance (scored from brief verbal descriptions in response to each face) for Respond, Baseline, and Suppress pictures in Depue et al.'s task

presented with the Faces as cues, and asked to either retrieve the associated scene or to suppress it. On the final test, participants were given each of the faces, and asked to provide a brief one sentence description of the associated scene, enough to allow independent judges to assess whether it had been retrieved. Independent raters then scored these verbal descriptions as to whether they signaled the appropriate scene. Depue et al. found that subjects showed a negative control effect (9\%) for suppressed scenes, and a positive control effect for retrieved scenes $(9 \%)$ for a total control effect of $18 \%$ (see also Depue et al., 2006). The demonstration of negative control effects across this range of stimuli indicates that the effect is not specific to verbal items, but affects episodic traces more generally.

\section{Generalizes to Emotional Memories}

It retrieval suppression is to provide a model of motivated forgetting, negative control effects should occur for traces with emotional content, particularly memories about negative experiences. A number of studies have found negative control effects in which the trace to be suppressed was negative, including negative words (Depue et al., 2006; Hertel \& McDaniel, 2010; Joormann et al., 2005; Joormann et al.,2009; LeMoult et al., 2010; Kim et al., 2007; Lambert et al., 2010. Murray, Muscatel, \& Kensinger, 2011) and negative pictures (Depue et al., 2007).

A particularly nice illustration comes from the study by Depue et al. (2007) discussed earlier, in which participants showed a $9 \%$ negative control effect in their ability to recall scenes in response to faces (Fig. 7). Importantly, all of the scenes used in this study were drawn from the International Affective Picture system (IAPS), and were highly unpleasant in character. Photographs included images of car accidents, people with injuries, and other unpleasant subject matter. These studies demonstrate that emotionally charged and unpleasant experiences are not immune to the effects of retrieval suppression, as one would expect if the process helps to control unwanted memories. 
Although it is clear that emotional materials can be suppressed, it is less clear whether negative memories are more, less, or equivalently suppressible, compared to emotionally neutral or positive experiences. On the one hand, people may be motivated to suppress unpleasant items, resulting in larger negative control effects than would be observed for neutral materials. On the other hand, emotional experiences may be intrinsically more intrusive, and so might be difficult to suppress. Studies comparing the ability to suppress negative and neutral materials have yielded inconsistent results. Some have found that negative traces show larger negative control effects than do neutral or positive traces (Depue et al., 2006; Joormann et al., 2005; Lambert et al., 2010). Other authors have found that negative memories show smaller negative control effects than do neutral ones (Hertel \& Gerstle, 2003; Marx, Marshall, \& Castro, 2008; Nørby, Lange, \& Larsen, 2010). Other authors have reported similar impairment on neutral, negative, and positive items (Hulbert, Anderson, \& Kuhl, in preparation; Murray, Muscatell, \& Kensinger, 2011).

It is unclear what underlies these variations. One explanation lies in the manner in which emotional stimulus sets are designed, and, in particular, whether neutral and negative stimuli are matched on variables other than valence and arousal that might vary across negative and neutral materials. Negative materials, for example, tend to come from a small set of categories that evoke strongly negative responses, including stimuli that concern death, disgust, anger, fear, and violence. Moreover, negative emotion words are generally more abstract, on average, than neutral words. In contrast, neutral words derive from a greater diversity of categories, and so may, on average, have greatly reduced inter-stimulus similarity. If negative items have higher inter-relatedness, one can no longer assume that performance on Respond and Suppress items is independent, as actively thinking about some pairs (e.g., HillDeath) may make it harder to suppress highly related pairs (Lake-Kill) (see, e.g., Goodmon \& Anderson, 2011 for demonstrations of how semantic relatedness insulates items from inhibition in retrieval-induced forgetting). Because neutral pairs will be less related, they would not suffer from this difficulty. Some of the variability across studies in the relative ease of suppressing neutral and negative materials surely arises from variations in the control of these factors. Supporting this view, Hulbert et al. (in preparation) demonstrated that when negative and neutral words are matched on inter-item similarity, concreteness, frequency, length and other variables, the negative control effects are similar for emotional and nonemotional stimuli.

Nevertheless, it would be desirable to experimentally manipulate inter-pair relatedness to verify that this factor modulates the negative control effect.

\section{Effects of Retention Interval on the Effect are Unclear}

Only two studies have examined whether the negative control effect changes over time, and these studies have yielded somewhat inconsistent findings. Norby et al. (2010) found a significant negative control effect for neutral materials on an immediate test (13\%), but no negative control effect when those same participants were 
brought back to the laboratory and retested on the same items one week later $(+1 \%$ facilitation). In contrast, Hotta and Kawaguchi (2009) found significant negative control effects on an immediate test (20\%) and a re-test on those same items conducted after $24 \mathrm{~h}(10 \%)$.

The reliable effect observed by Hotta and Kawaguchi after $24 \mathrm{~h}$ indicates that the negative control effect is not merely a momentary deficit that dissipates shortly after retrieval suppression has ended. Nevertheless, there is some indication that this effect may dissipate. Both studies both show the negative control effect to be reduced after an extended delay, even though it remained significant in one. Perhaps the full release observed by Norby et al. arose simply because they waited a week to retest the items, whereas Hotta and Kawaguchi only waited one day. Unfortunately, one cannot be confident that the reductions in the negative control effect reflect its dissipation with time. Both studies used a test-retest method that complicates interpretation. For example, successful retrieval practice improves an item's later retention, and, moreover, retards the rate of forgetting over longer retention intervals (e.g., Karpicke \& Roediger, 2008), particularly when the retrieval is difficult. Perhaps the initial test strengthened items that were retrieved, and differentially so, depending on whether retrieval was difficult (Suppress items) or easy (Baseline items). If so, one might expect this initial retrieval to create items with differing forgetting rates, with initially retrieved baseline items being forgotten more quickly than initially retrieved Suppress items. Moreover, the first test may have released inhibition for some items, creating an underestimate of the inhibition that might have occurred on a delayed test had no initial test happened. A purer test of the effects of delay on the negative control effect is clearly warranted.

It is worth noting that the effects of delay on other inhibitory phenomena are similarly inconsistent. For instance, in research on retrieval-induced forgetting, some authors have reported full recovery from inhibition after a day or more (Chan, 2009; MacLeod \& Macrae, 2001; Saunders \& MacLeod, 2002), concluding that the effect is transient, whereas other authors have reported inhibition after $24 \mathrm{~h}$ (Ford, Keating, \& Patel, 2004; Conroy \& Salmon, 2005; Conroy \& Salmon, 2006; Garcia-Bajos, Migueles, \& Anderson, 2009; Storm et al., 2006; Racsmány, Conway, \& Demeter, 2010; Tandoh \& Naka, 2007). Indeed, Garcia-Bajos, Migueles, and Anderson found, using an eyewitness memory video, retrieval-induced forgetting after a week that was significant and undiminished (Garcia-Bajos et al., 2009). Notably many of these demonstrations do not suffer from the repeated testing problem described above. Here again, what determines whether inhibition dissipates or persists remains unclear. One possibility is that persisting effects are more likely when the later retrieval of the suppressed representation cannot easily be supported by pre-existing semantic knowledge, and is thus more strictly episodic in character. This might arise for example, if episodic representations are more disrupted by inhibition (Anderson \& Spellman, 1995; Anderson, 2003). A parallel possibility may also exist with the negative control effect.

Although evidence for dissipation of the negative control effect is theoretically interesting, one must be cautious about generalizing conclusions about durability in these studies to real life cases of memory control. For instance, even if the negative 
control effect dissipates after a week in the conventional Think/No-Think paradigm, one must bear in mind that the effects induced by this procedure reflect the efforts of participants in a single brief session, with the total duration of suppression lasting only about $1 \mathrm{~min}$ (12 repetitions, $4 \mathrm{~s}$ each). In contrast, real cases that require memory control are likely to entail more instances of suppression, distributed over longer time intervals (in some cases, perhaps years), and implemented by a highly motivated person. One cannot be sure how the impact of distributed efforts to suppress accumulates over time, and whether effects of greater duration are possible. Nevertheless, evidence from the few studies that have been conducted suggests that memories, once suppressed, can later be recovered. This suggests that it may be possible to forget, and later recover a suppressed experience, under the right conditions.

\section{The Negative Control Effect Sometimes Does Not Occur}

Although, the negative control effect has been replicated many times, sometimes no reliable effect is observed even though it would be expected (e.g., Bulevich et al., 2006; Bergström, Velmans, de Fockert, \& Richardson-Klavehn, 2007; Mecklinger, Parra, \& Waldhauser, 2009; Hertel \& Mahan, 2008; Hertel \& Calcaterra, 2005). For instance, Bulevich et al. conducted three experiments with variants of the Think/ No-Think paradigm that closely paralleled earlier studies and observed 3\%, 4\%, and $1 \%$ negative control effects on the Same probe test, and similarly small effects on the Independent Probe test. Mecklinger, Parra and Waldhauser found a 1\% Same Probe effect and a 5\% Independent Probe effect. Hertel \& Mahan observed 4\% negative control effects in two samples, and Hertel \& Calcaterra found no negative control effects in their uninstructed group. A question arises as to why negative control effects failed to emerge in cases like these.

There are likely to be several reasons why null effects sometimes occur. First, some are explained by subjects' noncompliance with the Suppress instructions. Unless one takes care to disguise mention of "memory" and "testing," some participants willfully disregard the instructions and use the cue presentation as an opportunity to intentionally retrieve and rehearse the suppression item. Moreover, even when participants think they are complying with Suppress instructions, they occasionally "just check their memory to still see if they know the answer" either during or after the Suppress trial has ended. When subjects are non-compliant, one cannot reasonably expect to see memory deficits for Suppress items, as subjects are not faithfully suppressing retrieval. We address this issue by eliminating all mention of memory (in the procedure, consent forms, sign up sheets, etc.), and emphasizing that the experiment is a study of attention. We also administer a post-experimental rating scale to quantify noncompliance. Other authors may not take these precautions, and so may have elevated rates of non-compliance.

To illustrate the effects of compliance on the negative control effect, consider the study by Hertel and Calcaterra (2005) that manipulated whether participants were given thought substitutes. Hertel and Calcaterra administered the non-compliance 


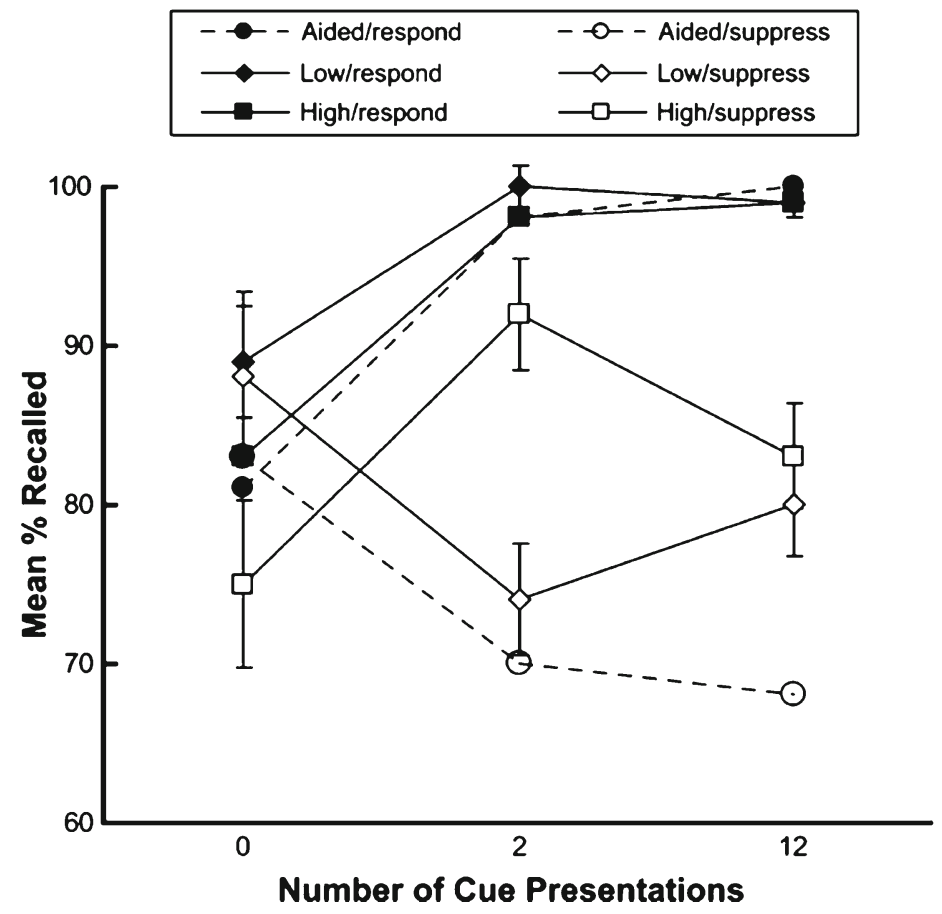

Fig. 8 Final recall for Respond and Suppression items as a function of the number of repetitions, from Hertel \& Calcaterra, 2005 (Reprinted with permission, copyright $@ 2005$ Springer Science + Business Media). Scores for noncompliance with suppression instructions (low vs. high) were derived based on the summed ratings of a strategy questionnaire administered after the final test phase. These scores were used to conduct a median split of subjects into low and high non-compliance, matching for counterbalancing. Of importance here is the difference between the low/suppress group and the high/suppress groups. The low/suppress (low noncompliance) group showed a significantly larger negative control effect than did the high/suppress (high noncompliance) group, who actually showed marginally significant facilitation

questionnaire we devised, and divided their uninstructed group into high and low compliance groups, matching on all counterbalancing dimensions. The results of this analysis are reported in Fig. 8. The pattern is what one would expect: noncompliant subjects who reported intentionally thinking of Suppress items showed progressive facilitation above baseline (+7\%) with increasing repetitions for Suppress items; by contrast, compliant subjects who honestly attempted to suppress showed a negative control effect $(-8 \%)$. Thus, the overall lack of a negative control effect in the uninstructed group reflects the prevalence of non-compliance, and the consequent canceling of negative control effects with retrieval practice benefits.

We do not think that compliance is the sole issue, however. Some null effects may arise because subjects do not remain vigilant for the full 30 min they are required to do so. Because controlling retrieval is effortful, fatigue is a substantial factor that undermines vigilance, and we know, from post-experimental measures, that subjects' efforts wane over blocks. Indeed, a drop in retrieval suppression 
performance with sustained effort would be predicted based on research on ego depletion, which consistently finds self-control deficiencies after a sustained period of control (Baumeister, Bratslavsky, Muraven, \& Tice, 1998; see Hagger, Wood, Stiff, \& Chatzisarantis, 2010 for a meta-analysis). If subjects lose vigilance in later blocks, some Suppress items may intrude, and, in effect, be given retrieval practice, countering impairment that may have arisen for other successfully suppressed items. As such, variations in whether researchers give rest breaks, the procedure is run in early morning, or experimenters keep participants motivated over blocks may account for some null negative control effects. This would make sense because forgetting should not arise if participants don't make an effort to suppress retrieval. However, although the negative control effect is sometimes not observed, it is clear from the combined analyses presented in Fig. 3 that the negative control effect is the typical pattern. Moreover, the total control effect, as far as we know, has always been found in published studies, showing that retrieval suppression generally terminates the benefits of seeing reminders. This suggests that effective intentional retrieval control is the rule.

\section{Trial Duration}

Several other variables have been proposed to affect the magnitude of the negative control effect, though these effects require further replication. For instance, Lee et al. (2007) hypothesized that longer duration trials result in weaker negative control effects than shorter ones. By this hypothesis, longer duration trials provide more opportunity for control to fail, and for the item to intrude, facilitating retention. Consistent with this, they observed that a group of participants given $3 \mathrm{~s}$ trials showed a $10 \%$ negative control effect, whereas a group given $5 \mathrm{~s}$ trials showed a $0 \%$ negative control effect. This result is consistent with the possibility that having to sustain cognitive control for longer durations may pose a substantial challenge.

Although this finding is intriguing, we unfortunately cannot disentangle whether the effect reflects the duration of individual trials or to the total duration of the think/ no-think phase, which is necessarily confounded in their between subjects design. In the latter case, the smaller effect may reflect fatigue affecting later trials, as discussed in the preceding section. Nevertheless, if this finding reflects trial duration, it would indicate that inescapable cues provide especially strong challenges to cognitive control, requiring sustained efforts over time to achieve full control over unwanted memories.

\section{Effects of Test Type}

Although most studies have used cued recall, several have examined whether negative control effects occur on tests that provide the item intact for recognition or on other indirect tasks. In a study by Kim and Yi (2008), participants studied worddrawing pairs and then performed the Think/No-Think task with words as cues. 
Kim and Yi tested retention on indirect tests, including perceptual identification of the picture under conditions of extremely rapid $(35 \mathrm{~ms})$ presentation (Experiment 1$)$, and identification of pictures in perceptual noise (Experiments 2 and 3). In all cases, twelve suppression attempts impaired performance on these tasks compared to baseline. For instance, participants identified only 33\% of the pictures after 12 suppression attempts, compared to $44 \%$ in the baseline condition. Experiments 2 and 3 showed that when the test introduced visual noise, participants needed to have more noise eliminated from the picture before they could identify it if it had been suppressed. Interestingly, these suppression effects only occurred for intact, but not mirror-reversed stimuli, indicating that suppression had affected perceptual representations of the pictures.

Similar effects can also be observed on explicit tests of recognition, although there are inconsistencies. For instance, Waldhauser, Johanssen, \& Lindgren (submitted) observed a negative control effect on item recognition $(6 \%)$ with words. Recently, in unpublished work, we have observed a reliable 9\% negative control effect on item recognition with verbal items that is qualitatively similar in magnitude to the cued recall effect. However, Tomlinson et al. (2009) reported a small but non-reliable negative control effect on item recognition (2\%), even when negative control effects were observed in cued recall. It must be noted, however, that their recognition test was administered after their recall test, and overall recognition performance was close to ceiling, either of which potentially complicates interpretation. Nevertheless, further study would be helpful to clarify the conditions under which this memory deficit occurs.

\section{Correlations with Stop Signal Inhibition}

Suppressing retrieval may be related to the capacity to override prepotent motor responses. One widely used tool that quantifies the ability to stop motor actions is the Stop-Signal paradigm, which measures the speed with which one can terminate an initiated motor action when a (typically) auditory signal is given (Logan, Cowan, \& Davis, 1994). Using this measure of motor inhibition, Depue et al. (2010) observed a negative correlation between stop signal reaction time on a motor response task $(-.58)$ and the proportion of emotionally negative pictures items successfully forgotten after retrieval suppression. Thus, the faster people were able to stop an initiated motor action, the more memory inhibition they showed, indicating that motor stopping speed is related to retrieval suppression ability. Moreover, performance on both retrieval suppression and stopping tasks correlated with engagement of right lateral prefrontal cortex during retrieval suppression, suggesting that the mechanisms underlying performance on these tasks may be related. In addition, as will be discussed later, Mecklinger et al. (2009) found that the N2, an electrophysiological component related to cognitive control was elevated for Suppress items and also for motor stopping trials on a stop signal task done with the same subjects one year later. Interestingly, despite that interval, the N2 increase in each stopping task was correlated. Although more work needs to be done to firmly establish the relationship 
between these capacities, the behavioral and neural similarities of these tasks suggests that the response override model of retrieval suppression may be correct (Anderson \& Weaver, 2009).

\section{Population Differences in the Negative Control Effect}

When one conducts research on retrieval suppression, one cannot help but be impressed at the variability in the negative control effect across participants. Whereas the average effect may be $7-10 \%$, it is not uncommon to find subjects who are exceedingly good at it (showing 50-60\% negative control effects), and also to find subjects who are exceedingly bad (showing substantial reversals of the effect). Correspondingly, the variability in the perceived difficulty of the task is striking, with some participants proclaiming the task to be trivial, and others, with equal insistence, stating that it was impossible for them to ever exclude memories from consciousness. This variability hints at important individual differences in the ability to control unwanted memories that are obscured when we average over large numbers of subjects with varying characteristics. This variability may be important in predicting which people may be vulnerable to intrusive memories in the aftermath of traumatic experience. The question naturally arises as to what causes this variability.

One key hypothesis about the variability in memory control ability is that it originates from broader deficits in the inhibitory control of action and thought. A growing body of research has examined this executive deficit hypothesis (|Levy \& Anderson, 2008) in populations hypothesized to have deficits in inhibition. The motivation for studying these populations is often twofold. First, to the extent that prior research indicates that the population has diminished control, a deficit in retrieval suppression would suggest that it engages more general mechanisms, as hypothesized in our response override framework. Second, documenting a deficiency in memory control indicates vulnerability to intrusive memories that may be of clinical significance. Research on memory control has focused on effects of age, attention deficits, depression, and traumatic experience as sources of individual differences.

\section{Aging Effects}

A number of investigators have proposed that cognitive control declines with age, and that inhibitory control declines in particular (e.g., Hasher \& Zacks, 1988; see Lustig, Hasher, \& Tonev, 2001, 2010b, for a review). If negative control effects reflect the action of a general inhibitory control mechanism, one should find that older adults are less able to suppress unwanted memories, and show reduced negative control effects. To date, there are have been two studies, and the data provide mixed support for this hypothesis. For instance, Anderson, Reinholz, Kuhl, and 
Mayr (2011) conducted two experiments in which they manipulated the number of suppression trials for younger and older adults. Anderson et al. predicted that older adult should show reduced negative control effects, particularly on the independent probe test, which eliminates the interference component of the effect. Consistent with this prediction, younger adults showed reliable negative control effects on the independent probe test (8\% and 7\% in Experiments 1 and 2, respectively), whereas older adults did not (4\% facilitation above baseline in both Experiments). In contrast, on the Same Probe test, older and younger adults showed negative control effects that did not differ, consistent with a role of interference on that test.

A different finding was observed by Murray, Muscatel, \& Kensinger (2011), who also manipulated the emotional valence of to-be-suppressed items. These authors found reliable negative control effects on the Same Probe test that did not differ by age in several experiments, consistent with Anderson et al.'s findings. Moreover, in the one experiment with older adults they conducted using independent probes, older adults did not show negative control effects on neutral items, consistent with Anderson et al. (2011). However, for memories with positive or negative emotional valence, older adults showed reliable negative control effects on an independent probe test. On the face of it, these results indicate that, at least for emotionally valenced materials, inhibitory control may be sufficiently preserved to support reliable negative control effects, at least in this sample. However, when all of the independent probe data is considered in the aggregate across all published studies (weighted average), young adults $(\mathrm{N}=104)$ show a 9\% negative control effect, whereas older adults $(\mathrm{N}=81)$ show a $2 \%$ effect. Thus, the overall tendency is for there to be an inhibitory deficit in older adults. Nevertheless, it is clearly desirable to identify why in some cases older adults show negative control effects.

\section{Developmental Effects}

Cognitive control improves across late childhood and early adolescence, and a number of investigators have argued that this development reflects increasingly effective inhibitory control (e.g., Harnishfeger \& Pope, 1996; Wilson \& Kipp, 1998). If so, one should observe a developmental progression in the ability to suppress unwanted memories with negative control effects emerging in middle childhood (10-12 year of age). This was tested by Paz-Alonso et al. (2009) who compared negative control effects across 8-9 year olds, 10-12 year olds, and young adults. Strikingly, the negative control effect increased with age, being absent for the youngest group, but present in middle childhood and in adulthood on both the Same Probe and Independent Probe tests. There was a continuous improvement with age, within childhood in the size of the negative control effect. Of interest, this negative control effect during middle childhood years occurred against a backdrop of overall improvements in declarative memory over this age range. Recently, Ogle and PazAlonso's (in preparation) have replicated this developmental trend of negative control effect improvement during middle childhood years with neutral non-arousing materials as well as with negative arousing word stimuli. These findings are consistent 
with recent evidence suggesting developmental progressions in inhibitory control as reflected in retrieval-induced forgetting (Aslan \& Bäuml, 2010) (see also, our later section on neural mechanisms, for a developmental fMRI study of retrieval suppression).

\section{Attention Deficit Disorder}

One prominent theory attributes symptoms of attention deficit disorder to impaired attentional control, and in particular, diminished inhibitory control (Adams, Derefinko, Milich, \& Fillmore, 2008; Barkley, 1997; Nigg, 2000, 2001; Quay, 1997). People with ADHD are less able to suppress prepotent motor responses in tasks such as the Go/No-Go and the Stop-Signal task (Oosterlaan, Logan, \& Sergeant, 1998), and, moreover, do not effectively engage right lateral prefrontal cortex in support of motor response inhibition in those same tasks (Booth et al., 2005; Tamm et al., 2004; Casey et al., 1997; Rubia et al., 2005). If retrieval suppression engages response override mechanisms, people with attention deficit disorder may show smaller negative control effects, and have difficulty controlling unwanted memories. Consistent with this possibility, Depue et al. (2010) observed reliably larger negative control effects for age matched controls (9\%) than for adults with ADHD $(0 \%)$ when people tried to suppress aversive photographs. Similarly, participants with ADHD show diminished retrieval-induced forgetting when tests control the influence of associative blocking (Storm \& White, 2010). These findings support the hypothesis that common functional systems may underlie memory and motor response suppression.

\section{Depression}

Depression is accompanied impaired memory and attention, and also a tendency towards ruminations about sadness. Several authors have proposed that depression diminishes cognitive control, making control over negative thoughts and feelings difficult (e.g., Hertel, 1994, 1998; Joormann, Yoon, \& Zetsche, 2007). Four studies have examined whether diminished memory control accompanies mild or clinical depression (Hertel \& Gerstle, 2003; Joormann et al., 2005, 2009; Hertel \& Mahan, 2008). All four report that participants with either mild (Hertel \& Gerstle, 2003) or major depressive disorder (Joormann et al., 2005, 2009; Hertel \& Mahan, 2008) show no negative control effect or a reversal of it, with recall of Suppress items improving as a function of repetition. These effects have been observed for positive and negative materials. The exception to this finding is a report of a substantial negative control effect for negative words in major depressive disorder, even though suppression of positive words was impaired (Joormann et al., 2005).

Although these findings are consistent with a deficit in memory control, additional work should be done to establish that this reflects an inhibition deficit. For example, no study has yet examined whether negative control effects can be 
observed on independent probe tests, which provides a cleaner assessment of whether inhibitory function is intact. Nevertheless, these findings are highly suggestive and are also consistent with a clinically relevant deficit. Consistent with this, the total control effect is correlated with scores on the Rumination on Sadness Scale (Hertel \& Gerstle, 2003), suggesting that a clinically relevant capacity is being measured. Moreover, this work has established extremely useful findings indicating that thought substitution can be used to improve control over unwanted memories (20-30\% negative control effects) even in major depressive disorder (Joormann et al., 2009).

\section{Effects of Trauma Frequency}

The more one practices cognitive or motor skills, the better one's performance. Perhaps this principle extends to retrieval suppression. According to this plasticity hypothesis, people with more experience at memory control might be better at suppressing unwanted memories and show larger negative control effects.

To get at this issue, Hulbert, Kuhl, \& Anderson (2011) examined the negative control effect in people who, prior to college, had few or many traumatic experiences. The frequency of such experiences was assessed with the Traumatic Experience Scale (Goldberg \& Freyd, 2006), which measures a broad spectrum of traumas, including accidents, disasters, violence, sexual assault or abuse, emotional abuse, and death of important people. In one experiment, participants were divided into groups based on their responses to this scale, administered after the Think/ No-Think task. In a second one, participants were prescreened as part of a course requirement, and we selected people with higher and lower scores. In both studies, we found larger negative control effects in people with more traumatic experiences, particularly when measured with an independent probe test. This advantage occurred for both negative and neutral words, and occurred even when (a) participants were offered money for right answers on the final test, and (b) experimenters and their supervisors were blind to the trauma status of subjects during the administration of the experiment and coding of the data.

The foregoing findings support the view that retrieval suppression ability is not fixed, but rather exhibits important plasticity. This plasticity raises the prospect that people suffering deficits in memory control may be able to improve mastery over intrusive experiences with proper training. The improvements may derive from strengthening an existing ability for retrieval suppression, or, instead, the development of adaptive strategies that improve forgetting, as illustrated by Joormann et al. (2009). It must be noted however, that a better demonstration of plasticity would involve randomly assigning participants to conditions and establishing a training effect, which remains to be done. Nevertheless, the fact that traumatic life experience predicts the negative control effect in the laboratory suggests that it measures mechanisms that may be engaged in everyday life. This suggests that the negative control effect provides a good model of motivated forgetting outside the laboratory. 


\section{Psychogenic Amnesia}

One intriguing study reported the memory control abilities of a psychogenic amnesic patient P.P., who suffered profound loss of his personal history at the age of 32, despite no evidence of brain damage or dysfunction (Tramoni et al., 2009). P.P. had a complete loss of autobiographical memory, but nevertheless had intact new learning ability, normal executive control function, and a higher than average IQ. P.P.'s memory control ability was examined with the version of the Think/No-Think task used by Anderson et al. (2004) to see whether he might have particularly large negative control effects, compared to control subjects. P.P. had no difficulty learning the pairs to the $60 \%$ criterion, and showed baseline and Respond item performance that was nearly identical, if not slightly higher than that exhibited by the group of 12 control subjects.

Strikingly, however, P.P. exhibited a $40 \%$ negative control effect on the same probe test, and a $60 \%$ effect on the independent probe test. Whereas he showed very high baseline performance (90\% ad $80 \%$ on the Same and Independent Probe tests), he showed extremely low recall of Suppression items $(50 \%$ and $20 \%$ on the same and independent probe tests). This was appreciably larger than the control subjects who showed a $7 \%$ negative control effect on the Same Probe test and a $10 \%$ effect on the independent probe test, typical of most studies. The authors concluded that P.P. appears to exhibit "hyper-suppression," which they speculate was triggered in response to the trauma that led to his psychogenic amnesia, and which may partially contribute to it.

Although it is difficult to know what to conclude from a single case study such as this one, and it is unclear how the putative hyper-suppression process might lead to involuntary forgetting of personal life experiences, this study is intriguing in its linkage of the negative control effect to a real life case of psychogenic amnesia. Nevertheless, further work needs to be done to establish whether other cases of psychogenic amnesia might be accompanied by hyper-suppression (see a related case in our later discussion of neural mechanisms), and to clearly articulate the mechanisms by which this might occur.

\section{Summary of Evidence for Retrieval Suppression}

As the foregoing review illustrates, people clearly can control retrieval, as indexed both by the total control effect and the negative control effect. Nearly every study conducted on retrieval suppression shows a total control effect, indicating that reminders do not intrinsically improve accessibility to related memories; rather, whether one benefits from reminders depends upon ones intentions and motivations, and whether those lead to the engagement of processes that shut down retrieval and terminate the normal benefits that would be expected by reminders.

The negative control effect indicates that retrieval stopping is accomplished by one or more processes that disrupt retention of the suppressed trace. The negative 
control effect has been replicated widely and, in the aggregate, yields clear evidence that retrieval suppression causes memory disruption, even after as little as 30-60s of suppression (12-16 repetitions of $3 \mathrm{~s}$ each). The negative control effect is likely to be multiply determined, with inhibition and associative interference contributing, depending on strategy and test type. Both thought substitution and direct suppression without thought substitutes induce negative control effects. Inhibition is most evident on the independent probe test, which is related to individual differences in inhibitory control and this effect may be larger in people with more traumatic experiences. The negative control effect generalizes to non-verbal and emotional stimuli, and can be found on recall and recognition tests. Importantly, the negative control effect is related more generally to the ability to override prepotent responses, including motor actions. Collectively, these findings provide a promising model for understanding the specific cognitive mechanisms that may underlie people's ability to control unwanted memories.

\section{A Neurobiological Model of Motivated Forgetting}

Response override provides a useful model of how motivated forgetting may occur. When people override retrieval, it impairs memory and does so, in part, by inhibiting the unwanted trace. Thus, motivated forgetting in real life circumstances may arise when people control unwanted memories by engaging systems that suppress overt action. However, although our functional analysis is a useful beginning, a more complete model of motivated forgetting would entail an understanding of its neural substrates. In this section, we discuss our efforts to build a neurocognitive model of memory suppression.

In our model, stopping retrieval is similar to stopping a motor action, except for the nature of the thing being stopped. If so, a common underlying network may be involved in implementing both types of stopping. Fortunately, a lot is known about the neural mechanisms of motor stopping, providing hypotheses about how memory stopping might be done. For example, in humans, imaging studies of motor suppression have shown that response override is associated with a network of controlrelated regions, including both ventrolateral and dorsolateral prefrontal cortex (e.g., Garavan et al., 2002; Menon et al., 2001; see Levy \& Wagner, 2011, for a metaanalysis). Correspondingly, animal research with the "go/no-go" task indicates that lesions to the lateral prefrontal cortex in monkeys impair their ability to stop a response (Iversen \& Mishkin, 1970). Even more striking, electrical stimulation of this same prefrontal region during a "go" response actually leads monkeys to terminate their motor response (Sasaki, Gemba, \& Tsujimoto, 1989). Thus the lateral PFC plays a critical role in stopping motor responses. Indeed, humans with lesions to the lateral prefrontal cortex show impaired stop-signal reaction time, indicating a substantial problem with stopping motor behavior (Aron et al., 2003). If retrieval suppression builds on mechanisms of response override, suppressing unwanted memories might also engage lateral prefrontal cortex. 
Although the stopping process may be similar, the nature of the representation that gets suppressed must vary between memory and motor stopping. Given that the goal of retrieval suppression is to suppress conscious recollection of a past experience, a process ascribed to the hippocampus (e.g., Squire, 1992; Eldridge et al., 2000), the hippocampus seems a likely candidate target to be affected. Thus, control-related regions in lateral prefrontal cortex may disengage hippocampal processes to prevent conscious recollection. This hippocampal modulation may be how we avoid catching our "mental cacti" and disrupt retention of unwanted memories. A number of studies have examined these neural hypotheses using functional magnetic resonance imaging.

\section{Neural Mechanisms of Retrieval Suppression: Basic Findings}

Anderson et al. (2004) addressed the foregoing hypotheses by using fMRI to identify brain regions that support retrieval suppression. Using a task similar to that described earlier, they scanned participants during the Think/No-Think phase. On a final test, they replicated the negative control effects on the Same and Independent Probe tests; they found that subjects recalled fewer suppression words than baseline words, indicating that participants had successfully suppressed their memories. To determine which brain regions were involved in retrieval suppression, they contrasted activation on "Suppress" trials and "Respond" trials. As predicted by the response override hypothesis, "Suppress" trials engaged control-related regions that overlapped strongly with those typically involved in stopping motor action, including lateral prefrontal cortex (both dorsolateral and ventrolateral regions), lateral premotor cortex, and anterior cingulate cortex (see Fig. 9). The strong engagement of control regions during suppression indicates that this goal is accomplished not by a passive failure to engage retrieval, but by engaging processes to prevent unwanted memories from coming to mind. Importantly, these findings support the idea that common brain regions may control stopping both unwanted memories and unwanted actions.

But what representation or system did retrieval suppression target to prevent retrieval? To examine this, Anderson et al. (2004) identified brain regions that were less active during Suppress trials compared to Respond trials. Importantly, there was a reduction in hippocampal activity bilaterally. This difference suggests that subjects are able to phasically regulate the activity of the hippocampus to engage or disengage the recollective process, as necessitated by the current goals of the rememberer. While this difference could be explained by increased hippocampal activity during "Respond" trials, it is also consistent with the hippocampus being down-regulated during suppression. Supporting the latter explanation, the degree of hippocampal activity during retrieval suppression was related to the size of the negative control effect observed on the later memory test (see Anderson et al., 2004, for a description of this relationship). The fact that hippocampal activity during suppression is correlated with below-baseline behavioral suppression suggests that subjects can strategically down-regulate mnemonic activity in the hippocampus to prevent conscious recollection and disrupt later memory. 


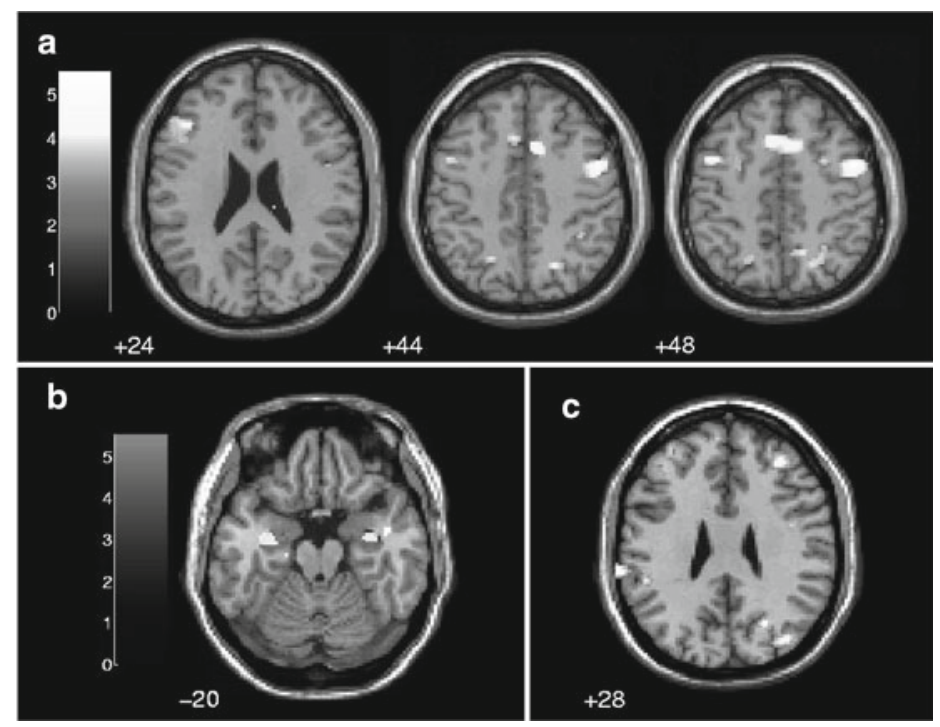

Fig. 9 TNT imaging data from Anderson et al., 2004 (Reprinted with permission from AAAS), showing that attentional control regions are recruited to control declarative memory retrieval. (a), lighter shaded areas are more active during retrieval suppression than during retrieval, and include areas generally associated with attentional control, including lateral prefrontal cortex (far left slice), anterior cingulate cortex (central area in several slices), lateral premotor cortex (rightmost 2 slices) and intraparietal sulcus (rightmost 2 slices, posterior left and right side). (b), lighter shaded areas are less active during suppression trials than respond trials, showing the hippocampus (middle, left and right side of image), a structure important for memory retrieval. (c), suppression-related areas in the DLPFC (anterior, right side) that predicted memory inhibition. It is hypothesized that the DLPFC exerts control over the hippocampus, reducing activation in the hippocampus, preventing memory retrieval from taking place and impairing retention

\section{Neural Mechanisms of Emotional Memory Suppression}

Although the study by Anderson et al. (2004) confirms the viability of response override as a model of motivated forgetting, the study used simple pairs of words without emotional content. Would the brain systems identified in that study be engaged by non-verbal materials that are aversive in character? Depue et al. (2007) studied this issue using the face-scene associations described earlier. Importantly, during the Think/No-Think task, when participants viewed a face and tried to suppress retrieval of the associated aversive image, they showed significantly more activation in right dorsolateral prefrontal cortex than during the Respond condition. As in the Anderson et al. study, activation in this region predicted individual differences in the negative control effect, with more activation associated with greater memory impairment. These findings converge with the view that the lateral prefrontal cortex is instrumental in disrupting retention via response override mechanisms.

Depue et al. observed several additional findings that may prove important to understanding retrieval suppression more broadly. First, suppressing retrieval of the 
aversive image reduced activation in right hippocampus below that observed on trials in which participants passively viewed a fixation cross on the screen, and were thus neither retrieving nor suppressing anything. This reduction is consistent with the view that retrieval suppression reduces hippocampal activity to disrupt conscious recollection, and broadly replicates earlier findings by Anderson et al. (2004) despite considerably more complex, naturalistic stimuli. Second, retrieval suppression significantly reduced amygdala activity, which fits with the broadly established role of this structure in emotion processing. Thus, whereas thinking of aversive pictures generated an emotional response reflected in elevated amygdala activity, suppressing retrieval reduced amygdala activity below passive fixation, suggesting that suppressing awareness of the unwanted memory pre-empted or attenuated unpleasant emotions that would have arisen had the participant recalled the unpleasant memory. Consistent with this view, other studies that have made suppressing unpleasant words difficult by extensive overtraining and by limiting suppression time ( $2 \mathrm{~s}$ instead of 4$)$, actually show elevated hippocampal and amygdala activity during retrieval suppression, possibly reflecting the unpleasant character of intrusions (Butler \& James, 2010). Taken together, these finding suggest an important role of retrieval suppression in regulating emotions after trauma: the more effectively memories can be inhibited, the less likely unpleasant retrievals will occur.

Finally, Depue et al. observed a progressive improvement in the intentional modulation of mnemonic activity in the hippocampus over blocks in the Think/No-Think phase. Whereas initial blocks showed suppression-related hippocampal activation elevated above a fixation baseline, increasing practice progressively reduced hippocampal activity below this baseline. In fact, the reduction in hippocampal activation during retrieval suppression in the final block predicted the negative control effect on the final test. Interestingly, Depue et al. suggested that practice may induce a qualitative shift in the networks that underlie retrieval suppression. In support of this, in early blocks, people did not show hippocampal modulation, but did show reductions in activity in visual cortex, together with engagement of ventrolateral prefrontal cortex. In later blocks, however, activation in dorsolateral prefrontal cortex became more prominent, as hippocampal reductions grew more effective. Depue suggested that this change with practice may reflect a shift in the mechanisms of control from ones that primarily prevent reinstatement of imagery associated with unpleasant scenes, to a DLPFC-hippocampal network that suppresses retrieval itself. This two-phase process needs further replication and formal testing. Nevertheless, these findings suggest that practice may make people more effective in engaging the neural systems that suppress retrieval, hinting at the viability of training interventions for people deficient in memory control.

\section{Electrophsyiological Indices of Retrieval Suppression}

A growing subfield in research on retrieval suppression seeks to develop electrophysiological indices of effective retrieval control using EEG. This research builds on a large body of evidence revealing a distinct signature of the subjective experience 
of recollecting a past event. ERP studies of recognition memory reveal a larger late positive component (LPC) over parietal scalp sites for older words compared to new distractor words. This component, which is sometimes referred to as the Parietal Episodic Memory (EM) effect (Friedman \& Johnson, 2000), appears 400-800 milliseconds after a target has been presented on a recognition test. Its amplitude increases with study-test repetitions when an item is consciously recollected (Johnson et al., 1998), is larger in association with those items rated as consciously remembered (Smith, 1993; Smith \& Guster, 1993), and is larger for words whose study context is correctly retrieved (Trott et al., 1999; Wilding et al., 1995; Wilding \& Rugg, 1996). If the parietal EM effect reflects conscious recollection, and if retrieval suppression terminates conscious recollection, one should find reduced effects during Suppress trials compared to Respond trials.

This prediction has been confirmed in numerous studies. For instance, using an adaptation of the standard Think/No-Think task, Bergstrom et al. (2007) examined whether retrieval suppression would modulate the parietal EM effect. They focused on two questions: (a) when examining only those items that were successfully learned initially and that could be successfully recalled on the final memory test (and thus were fully encoded and retained in memory), would suppressing retrieval modulate the parietal EM effect, and (b) how complete would the suppression of this effect be, in comparison to Suppress items that subjects never learned or recollected on the final test? Strikingly, Bergstrom et al. (2007) found that suppressing retrieval significantly reduced the parietal EM effect, though not quite to the level of never-learned Suppress items. This modulation of the parietal EM effect has been replicated repeatedly (Bergstrom, de Fockert, \& Richardson-Klavehn, 2009; Bergström et al., 2009; Hanslymar, Leipold, \& Bauml, 2010; Mecklinger et al., 2009). In a related finding, Bergstrom, Anderson, Buda, Simons, \& RichardsonKlavehn (submitted) found significant reductions of late parietal positivity with cues to richly encoded visual scenes, in response to retrieval suppression instructions. Interestingly, Bergstrom et al. (2007) also demonstrated that participants could, for the very same items, make the parietal EM effect come and go when instructions changed from retrieval to suppression.

Importantly, however, retrieval suppression does not always modulate the parietal EM effect, because it depends on the mechanism one uses to control retrieval. Bergstrom et al. (2009) compared the modulation of the parietal EM effect in people who controlled unwanted memories by thought substitution, with another group who used the direct suppression process discussed earlier. Importantly, subjects using direct suppression significantly modulated this component, as in prior studies, whereas subjects using thought substitution showed no modulation. If the LPC component truly indexes conscious recollection, as numerous studies have shown, this finding indicates that direct suppression is not accomplished by occupying awareness with alternative memories. Thought substitution presumably did not modulate this effect because recollecting thought substitutes itself would generate a parietal EM effect, making the two conditions indistinguishable. These data thus support the existence of qualitatively different approaches to memory control. 
Despite the evidence for control over conscious recollection manifest in the modulation of the parietal EM effect, the modulation of this component does not predict negative control effects (Bergstrom et al., 2007; Hanslmayr et al., 2010). Other electrophysiological effects have been successfully related to inhibition, however, at least in the context of direct suppression. Hanslmayr et al. found that asking people to suppress retrieval using direct suppression instructions significantly reduced positivity compared to Respond trials across right prefrontal and left parietal cortex late in the Suppress trial (from $1.6 \mathrm{~s}$ onwards). Importantly, the extent of this positivity reduction increased with the number of Suppress repetitions, but not the number of Respond repetitions, and its magnitude predicted the negative control effect (Fig. 10). Intriguingly, Hanslmayr also found a similar effect occurred in advance of Suppress trials during preparatory cues signalling the nature of the upcoming trial, suggesting that people can pre-engage control processes while preparing to shut down retrieval. These findings provide an electrophysiological window into the benefits of advanced preparation in enhancing negative control effects.

Finally, the evidence indicates that electrophysiological markers of cognitive control, such as the N2, are larger during retrieval suppression. This finding is significant because ERP studies examining motor stopping consistently report enhanced N2 components for stopping, such as the no-go N2 (Bekker et al., 2005; Bokura et al., 2001; Donkers \& van Boxtel, 2004; Eimer, 1993; Falkenstein et al., 1999; Garavan et al., 2002) and the stop signal N2 (Band \& van Boxtel, 1999; Logan et al., 1994; Schmajuk et al., 2006; van Boxtel et al., 2001; Ramautar et al., 2004). For example, Mecklinger et al. (2009) found significantly larger N2 for Suppress items in comparison to Respond items, and, importantly found this effect to be especially pronounced for Suppress items that were later forgotten on an independent probe test. Prior work on the motor No-Go N2 suggest that it may reflect either inhibition of the motor act itself (Kopp et al., 1996), detection of response conflict (Falkenstein, 2006) or both. The source of the effect is thought to be the anterior cingulate cortex, but the lateral prefrontal cortex has also been suggested (Lavric et al., 2004), consistent with brain areas involved in retrieval suppression. Bergstrom et al. (2009) also observed a similar, though earlier ERP negativity that predicted individual differences in later independent probe forgetting, and a later ERP negativity that predicted forgetting of individual Suppress items. Finally, Mecklinger et al. (2009) found that the magnitude of the N2 enhancement during stop-signal trials in a motor response suppression task was correlated with the enhanced N2 for Suppress trials. These findings provide converging evidence for the view that motor and memory stopping share underlying mechanisms.

Taken together, the foregoing findings build a compelling body of evidence that retrieval suppression can be indexed by electrophysiological markers that indicate whether people are successful at controlling mnemonic awareness. Moreover, these markers provide useful insights into the mechanisms that underlie retrieval stopping that converge with data from functional magnetic resonance imaging. 

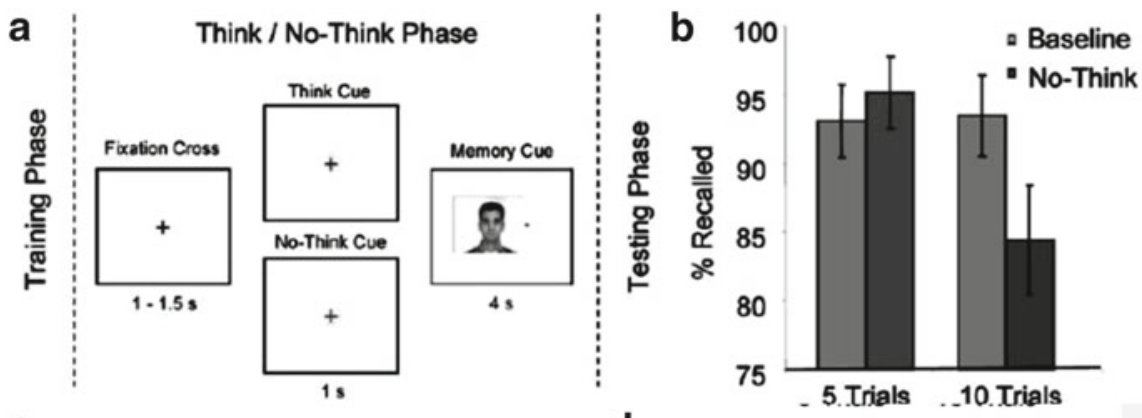

C
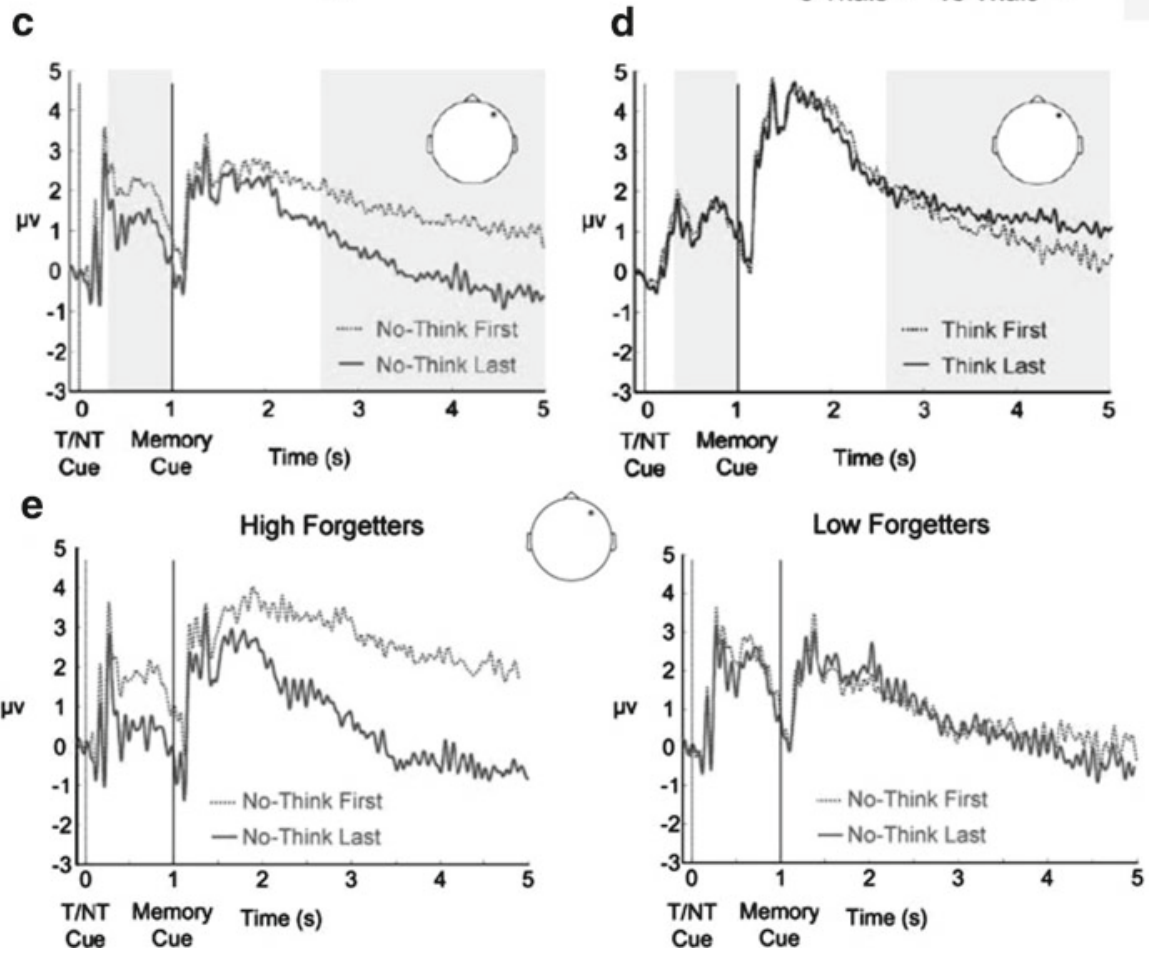

Fig. 10 Adapted from Hanslmayr et al., 2009. (a), Faces served as cues and words as responses. During the TNT phase, advance warning was given as to whether the trial would be a Suppress/ No-Think (red) or Think (green) trial. (b), Behavioral results. In the final cued recall test, forgetting (Baseline >Suppress) was absent after five Suppress trial repetitions, but was present after 10 Suppress trial repetitions. Error bars represent SE. (c), ERP waveforms for the first five (dotted line) and the last five (solid lines) Suppress trial repetitions for one representative electrode. Gray bars indicate the time windows during which significant differences emerged. (d), ERP waveforms for the first five (dotted line) and the last five (solid lines) Think trial repetitions for one representative electrode. (e), ERP waveforms for the first five (dotted line) and the last five (solid line) Suppress trial repetitions plotted for high and low forgetters, as deduced from a balanced median split on the forgetting scores (Adapted with permission, copyright (@) 2009 by the Society of Neuroscience) 


\section{Population Differences in Neural Mechanisms of Memory Suppression}

Several studies have compared the neural systems underlying retrieval suppression in neurologically normal adults to those engaged in other populations thought to have diminished or enhanced inhibitory control. In general, populations thought to be deficient in inhibitory control show diminished engagement of lateral prefrontal cortex in service of retrieval suppression.

\section{Attention Deficit Disorder}

Neuroimaging studies of attention deficit disorder have shown that individuals with ADHD do not engage right lateral prefrontal cortex as effectively as controls during motor response suppression (Booth et al., 2005; Casey et al., 1997; Depue et al. 2010; Rubia et al., 1999; Rubia, Brammer, Tonne, \& Taylor, 2005; Tamm, Menon, Ringel, \& Reiss, 2004). Based on the possibility that retrieval suppression may engage related response override mechanisms, Depue et al. (2010) used his face-scene think/no-think procedure to compare retrieval suppression in adults with and without attention deficit disorder to determine whether the former suffers deficits in retrieval suppression. As noted earlier, adults with ADHD showed smaller negative control effects than matched controls. Would these behavioural differences in retrieval suppression be reflected in the ability to modulate hippocampal activity? The imaging data revealed that matched controls engaged dorsolateral and ventrolateral prefrontal cortex during retrieval suppression, and significantly reduced activation in the hippocampus, replicating prior work by Anderson et al. (2004). Importantly, they showed a significant negative correlation between dorsolateral prefrontal cortex and hippocampus, and the size of this negative correlation predicted individual differences in forgetting of suppression items. In contrast to matched controls, ADHD participants failed to engage right dorsolateral prefrontal cortex during retrieval suppression and correspondingly failed to reduce mnemonic activity in the hippocampus. None of these interregional or behavioural correlations were observed for participants with ADHD. Interestingly, behavioural severity of ADHD symptomatology was related to the strength of the correlations between frontal and posterior cortical areas, with inattentive symptomatology predicting the magnitude of DLPFC-hippocampal negative correlations. These findings are consistent with the hypothesis that fronto-hippocampal modulation is a crucial neural mechanism underlying the suppression of unwanted memories and with the view that ADHD in part, reflects a deficit in inhibitory control. They further suggest that adults with attention deficit disorder should have difficulty with controlling intrusive memories. 


\section{Development of Retrieval Suppression}

The majority of research on retrieval suppression has focused on adults. A unique window on the systems essential to this ability, however, is provided by studying its development. As mentioned earlier, behavioural work has established an increasing efficacy at suppressing unwanted memories in middle childhood (Ogle \& Paz-Alonso, in prep; Paz-Alonso et al., 2009). Recently, Paz-alonso et al. (2011) have studied the neural basis of this shift. Forty-three participants from three age groups (fifteen 8-9 year olds, fourteen 11-12 year olds, and 14 young adults) were scanned as they performed the Think/No-Think task. Aggregating across all 43 participants showed robust engagement of right DLPFC and VLPFC during retrieval suppression, and a clear reduction in hippocampal activity during Suppress trials, consistent with the foregoing imaging studies by Anderson et al. (2004) and Depue et al. (2007). Moreover, activity in DLPFC was functionally related to activity in the hippocampus during retrieval suppression, indicating an interaction between these regions that helps to implement the process of retrieval suppression.

Comparing across age groups reveals several neural changes that characterize the development of memory control. First, whereas younger adults engaged lateral prefrontal cortex and posterior parietal cortex while effectively suppressing memory retrieval, 8-9 year olds did not effectively engage these regions. Second, in contrast to adults, children did not effectively modulate hippocampus activation during retrieval suppression. The 11-12 year olds showed an intermediate pattern of control and modulation. Finally, during retrieval suppression, the data revealed increased engagement of right posterior parietal cortex (BA 40/7) in adults compared to 8-9 year olds, together with a broad increase in functional connectivity between lateral prefrontral, cingulate, lateral posterior parietal, precuneus, and hippocampal regions, reflecting increasingly effective inter-regional communication with age. Thus, the emergence of the capacity to suppress unwanted memories reflects increasingly effective engagement of prefrontal cortex to control hippocampal activity, and, importantly, tighter coupling of the fronto-parietal-hippocampal network of regions involved in this process.

These data suggest that future work examining individual differences in memory control as well as the effects of practice on retrieval suppression would profit from looking at changes in inter-regional connectivity that might support superior control over unwanted memories. More generally, however, they suggest that retrieval suppression relies upon the emergence of cognitive control, supporting the view that this ability builds upon mechanisms of response override that are of broad importance in mental life. These findings also raise the question of how and whether the typical development of memory control might constrain the ability to suppress unwanted memories of abuse. Are the developmental time courses observed in these studies determined strictly by maturation, constraining when children can be expected to be effective at motivated forgetting? Or might early life challenges to memory control alter the pace of development of neural systems underlying memory control? 


\section{Psychogenic Amnesia}

The foregoing studies indicate that retrieval suppression engages lateral prefrontal cortex to reduce activation in the hippocampus as a means of controlling awareness of unwanted memories. Although the mechanisms observed in these studies could underlie real cases of intentional suppression, cases arise that do not, on their face, seem well characterized as being intentional. For instance, intensely stressful periods can sometimes induce psychogenic amnesia, in which the person may fail to remember large chunks of their personal experiences, forgetting who they are, even though their general knowledge and learning ability may remain intact. Could psychogenic amnesia sometimes be the result of spontaneous, involuntary application of the mechanisms identified here, as suggested by the earlier reviewed work of Tramoni et al. (2009)?

There is intriguing preliminary evidence that this involuntary control may happen. In a recent study, Kikuchi et al. (2010) studied two patients with dissociative amnesia. Both patients were well educated, and neurologically normal, and of normal intelligence, but both had undergone a recent stressful event or period of time that lead to extensive retrograde amnesia. For instance, Patient 1, a 27 year-old businessman exhibited focal retrograde amnesia for all events, people, and activities that took place in the 4.5 year period prior to the onset of his amnesia, even though he could recall experiences and people from before that period. Patient 2 presented a similar, but more extensive retrograde amnesia. No neurological abnormalities could be detected, and they appeared to remember all new experiences that happened to them after the onset of the amnesia, showing normal new learning.

Kikuchi et al. scanned these two patients as they viewed photographs of individuals taken from various periods of their lives. Specifically some photographs were of individuals they knew from the periods of their lives that they could still remember (recognizable photos), whereas others were from the window of retrograde amnesia (unrecognizable photos). Novel photographs of people unknown to the patients were also included. Participants simply judged whether or not they recognized each photograph as it was presented. The authors found that unrecognizable faces showed greater activation in dorsolateral and ventrolateral prefrontal cortex, compared to both recognizable and novel control faces, indicating the engagement of a network similar to that involved in with retrieval suppression. Moreover, these patients showed reduced hippocampal activation in response to the unrecognizable faces, compared to novel faces and recognizable faces consistent with the possibility that retrieval was being suppressed. Kikuchi et al. raised the intriguing possibility that extreme psychological distress may lead retrieval suppression processes to be engaged involuntarily in reaction to certain stimuli, over long periods of time, creating a pattern resembling that observed in laboratory studies of retrieval suppression. They acknowledge, however, reduced hippocampal activation for unrecognizable faces may reflect participants' lack of recollection of those faces, compared to recognizable items and that further work is required to establish their active down-regulation interpretation. Nevertheless these findings, 
with those reported by Tramoni discussed previously, illustrate how the response override and fronto-hippocampal modulation hypotheses provide an interesting and productive lens through which to view real life cases of motivated forgetting.

\section{Summary of Neural Basis of Memory Control}

Recent years have seen the emergence of a neurobiological model of motivated forgetting that integrates this otherwise controversial process with widely accepted and fundamental mechanisms for the control of human behavior and thought. Response override is a universally acknowledged function of cognitive control, and the neural mechanisms underlying it have received extensive study. Retrieval suppression can be profitably viewed as a special case of this process, in which the function to be stopped is episodic memory retrieval, and the targets of control are representations of episodic experience supported in part by the hippocampus.

Studies of retrieval suppression with simple words, and emotional pictures have now consistently shown that suppressing awareness of an unwanted memory engages lateral prefrontal cortical regions overlapping with those involved in response override to modulate neural activity in the hippocampus. These conclusions have received converging support from electrophysiological research, which has established several indices of retrieval suppression, and the likely engagement of response override. The extent to which response override mechanisms are engaged predicts forgetting of the suppressed trace, and individual differences in the function of these systems appears to be related to how well people control unwanted memories. There is even some preliminary indication that fronto-hippocampal modulation may provide a model of some cases of psychogenic amnesia. Much work remains to be done, however, to identify the precise relationship between memory and motor response suppression processes, to identify the pathways by which lateral prefrontal cortex exerts influence on the hippocampus, and to understand the nature of the disruption induced by hippocampal modulation. Taken together, this work specifies a useful and specific neurobiological model that reinforces the utility of the response override framework for understanding motivated forgetting.

\section{Building Retrieval Suppression as a Model of Motivated Forgetting}

Thus far, our discussion has focused on the variety of useful discoveries that have been made in current research on retrieval suppression. Despite these interesting successes, the case for retrieval suppression as a mechanism of motivated forgetting is in a relatively early stage of development (Anderson \& Levy, 2006). In the remainder of this article, we discuss important issues that remain to be addressed in future research to build a strong case for the role of retrieval suppression in motivated forgetting. 


\section{Generalization of Negative Control Effects to Ecologically Valid Memories}

Research on retrieval suppression was, at the outset, motivated by the observation that continuously confronting inescapable retrieval cues appeared to be related to subjective reports of forgetting for childhood abuse. The observation that retrieval suppression causes both total control effects and negative control effects illustrates one way in which this surprising relationship may come about. When reminders lead to recollections that one is motivated to not think about, inhibitory control mechanisms have a detrimental impact on the retention of the suppressed memory.

One must acknowledge, however, that there is a considerable gap in the nature of the memories studied in laboratory work and those present in these real cases. Simple pairs of words provide a useful beginning for understanding retrieval suppression, but ultimately research must examine whether such mechanisms can induce forgetting of complex, multi-modal, emotional events personally relevant to the rememberer. Does retrieval suppression "scale up" to real events? The work of Depue and colleagues demonstrating negative control effects with face-scene pairs moves in the right direction. Nevertheless, even these stimuli are relatively constrained. If it could be shown that negative control effects occur for naturalistic episodic experiences, and autobiographical memories, the case for the relevance of retrieval suppression would be stronger.

It must be emphasized that there are reasons why experimentalists begin studying a process with simple memory items like words and photographs. With materials like these, one can carefully control what is encoded, as well as what strategies or processes people bring to bear. The process of generalizing mechanisms to complex, less controlled stimuli is likely to present challenges and complexities that are difficult to anticipate. As such, patience and persistence is required to evaluate whether this generalization is possible. Nevertheless, the fact that the present work was motivated by a perplexing but similar pattern in self reports of memory for abuse suggests that it may be possible to span this considerable gap, given imagination and persistence.

\section{Persistence of the Negative Control Effect Over Time}

If retrieval suppression underlies some cases of motivated forgetting, it suggests that forgetting can persist over extended periods. To understand how this occurs, more work must be done to examine how long negative control effects last, and whether their durability provides a reasonable model of these phenomena. If negative control effects only lasted $5 \mathrm{~min}$, for example, one might question their relevance to motivated forgetting. However, if retrieval suppression can produce durable forgetting, or that forgetting can be sustained by other means, it would strengthen its relevance to motivated forgetting, and define the conditions under which these effects to occur. 
Most research on the persistence of negative control effects has focused on the conventional procedure without instructions about how to control memory. The findings thus far indicate that negative control effects may last somewhere between one day and one week. Several factors remain unexplored, however, that may influence the impact of suppression on long-term retention. First, one must consider the cumulative effects of suppression over many repetitions spread out over time and how this may affect the durability of forgetting. As noted earlier the cumulative time suppressing an item in the Think/No-Think procedure is between $45 \mathrm{~s}$ and a minute, all within a single half an hour session. Real cases of memory control are likely to involve more protracted efforts, spread out over months or longer. These situations differ in the amount of suppression and in its schedule. Distributed, recurring efforts at suppression may have more enduring effects on the negative control effect, much like distributed repetition of memory items has far bigger effects on retention than do massed repetitions. Thus, estimates of the durability of negative control effects based on a small number of suppressions within a session may underestimate the longevity of the effect in real life cases. Clearly, this distributed practice hypothesis needs to be tested.

Second, the relevance of present efforts to estimate the durability of negative control effects to real cases of motivated forgetting may be limited by the test methodology. All current tests of the durability of negative control effects ask people to explicitly recall unwanted memories on the final test, a situation unlikely to occur in real settings. As Hertel, Large, Dahl, and Levy (2011) aptly argue, it is unlikely that someone motivated to exclude an unwanted memory from awareness would turn around and try to retrieve that memory. Rather, upon encountering cues to the unwanted memory, they will, if anything, be biased away from any such retrieval, especially given their efforts to develop alternative thoughts in relation to reminders. Thus, according to this retrieval tendency hypothesis, a better estimate of the impact of suppression on the control of awareness, in real terms, would estimate the spontaneous retrieval of the unwanted memory in response to a cue, when no instructions are given to recall anything (e.g., providing the first thought that comes to mind; Hertel et al.). Perhaps people never recall the unwanted items spontaneously when given reminders and always manage to think of something else first. In practical terms, this accomplishes the goal of keeping the unwanted memory from awareness. Indeed, as discussed later, spontaneous retrieval of alternative associations provides one way of reinstating negative control effects.

Third, one must consider that estimates of the average durability of negative control effects, based on a large sample may not reflect the durability exhibited by people who are exceptionally effective at suppressing memories. As discussed shortly, insufficient attention has been given to studying the extremes of memory control, and the manner in which the impact of suppression in those participants may differ from the typical effect. Such extremes may emerge because of ability or expertise. Expertise at retrieval suppression may reflect superior strategies for retrieval suppression, or strengthening of cognitive control through extensive practice. Thus, a better understanding of the durability of negative control effects and their implications for real cases requires studying people who are especially good at it. 


\section{The Extremes of Motivated Forgetting}

The cases that started the recovered memory debate are ones in which people claim to forget disturbing experiences over many years. Many people find such cases difficult to believe because they defy the intuition that if something that unusual happened, we would remember it. Given these considerations, if some cases are real, they may indeed not be the norm. Perhaps the vast majority of people having such experiences would remember them, confirming the average person's intuition. If so, then cases in which abuse is truly forgotten might be extremes on a continuum, such that only people with strong cognitive control are capable of it.

If this control ability hypothesis is correct, there is an important disconnect in the relevance of the current laboratory approach to real cases. In laboratory studies, research focuses on the average negative control effect, collapsed over many people with widely varying control abilities. The characteristics of this sample are not representative of people likely to succeed at suppressing truly unpleasant and unusual memories. Arguably what research should be doing instead is trying to understand people who are hypereffective at suppression. Indeed, retrieval suppression need not be exceptionally powerful in all people. Rather, all it takes for retrieval suppression to be an excellent model of recovered memories is for a small fraction of people to be profoundly good at it.

One approach to conducting such research is to study individuals who recover memories of abuse after years of forgetting. Presumably if the abuse event can be corroborated as having truly occurred, individuals who forget may have better memory control abilities than people who also have corroborated abuse, but who always remembered it. If so, one might find larger negative control effects in such cases than in individuals who have had continuous memory of the abuse. Such an approach has been taken in related research by Geraerts and colleagues, who have studied thought suppression abilities in people with recovered memories (Geraerts, McNally, Jelicic, Merckelbach, \& Raymaekers, 2008). Interestingly, people who recovered memories of abuse spontaneously, outside of therapy do in fact show superior thought suppression capability, consistent with this hypothesis.

Another approach would be to identify people who show large negative control effects, and study them to determine whether or not have characteristics that would be consistent with either enhanced cognitive control in general, or particularly effective strategies. Might effective suppressors show superior performance on stop signal reaction time tasks or other measures of executive function? Might they show more effective engagement of lateral prefrontal cortex during retrieval suppression? The systematic characterization of effective suppressors may be extremely helpful in identifying characteristics of individuals who might be especially prone to be good at memory control. It would also be helpful to know the proportion of the population that is capable of extremely effective retrieval suppression. If only a small fraction of individuals is capable of hyper-suppression, this may explain why people often think that this level of control over one's memory seems implausible. 


\section{Conditions of Memory Recovery}

Surprisingly little work has focused on whether memories can be recovered, once they have been suppressed. To develop retrieval suppression as a model of motivated forgetting that can account for the forgetting of child abuse that is later accompanied by the recovery of the abuse, it would be helpful to explore whether recovery following suppression is possible, the conditions that trigger recovery, and the characteristics of memories that are recovered. If the conditions of recovery can be delineated in experimental work, it may help to understand when and how memories may be recovered in real cases.

Several conditions are likely to contribute to memory recovery, and it should be possible to clearly document these in laboratory studies. First, in general, the more times that one attempts to retrieve the same information, the more one recalls, even when one feels that one cannot recall any more, a phenomenon known as hypermnesia (Erdelyi \& Kleinbard, 1978; Payne, 1987). Moreover, even when overall recall does not increase with repeated retrieval, previously unrecalled items often get recalled on later tests (but are balanced by forgetting of previously recalled items), a phenomenon known as reminiscence. Although some work has already shown that hypermnesia is possible for to-be-forgotten items in the directed forgetting procedure (Goernert \& Wolfe, 1997; Goernert, 2005), no work has yet examined whether hypermnesia or reminiscense can also be found with retrieval suppression. This reminiscence hypothesis predicts that all or part of unwanted memories may be recoverable, given repeated efforts at retrieval, though, under such circumstances, one must also be concerned about the introduction of reconstructive errors that may distort memory (see, e.g., Henkel, 2004).

Second, the more cue information one provides, the more likely that retrieval may succeed, even if suppression has occurred. Although suppression ought to impair memory from a variety of cues, this does not mean that adding cuing information shouldn't help increase the chances that a suppressed memory can be recovered, at least in part. There have been elegant demonstrations of powerful cue-based recovery effects in experimental paradigms other than retrieval suppression (Smith \& Moynan, 2008), but research has not yet examined how varying the number of cues influences items suffering from negative control effects. One possibility is that providing cues for baseline and suppression items simply raises overall performance in both conditions, leaving negative control effects unaffected. Another possibility is that negative control effects may differentially benefit from cues, resulting in a "release" effect. Regardless of which pattern is observed, however, if additional cues help participants recall items that would have been forgotten, it would suggest that encountering related cues in everyday life should increase the chances of a suppressed memory being recovered. Whether some cues might be more powerful in eliciting recovery than others is also a question of interest. For instance, reinstatement of spatial or emotional context may be important.

Third, the passage of time itself may contribute to memory recovery, as assumed by current attempts to study the longevity of negative control effects. On this view, 
items suffering from negative control effects may undergo a gradual change in state over time that increases their accessibility. For instance, inhibition may gradually dissipate over time. The idea that time may be an important factor predicting the release of inhibition owes its conceptual heritage to research on spontaneous recovery from extinction in research on classical conditioning (Pavlov, 1927, Rescorla, 2004), and on analogous recovery effects in episodic memory research on retroactive interference (Underwood, 1949, Brown, 1976, Wheeler, 1995). The passage of time has also been proposed to enhance reminiscence and hypermnesia effects in repeated recall, even when participants are fully occupied with other tasks in between repetitions, in a phenomenon called incubated reminiscence (Smith \& Vela, 1991). Negative control effects may exhibit a similar release over time, though, as discussed previously, the issue needs further exploration.

Finally, the parameters that determine when a memory will be recovered may be different for real cases of motivated forgetting, if having a genuine motive for forgetting matters. For instance, reminders of an unpleasant experience such as abuse may be threatening during childhood, but as circumstances change and a person grows to be more self sufficient, secure, and independent of the abuser, feelings of threat that drive maintenance of memory control may subside. If the motive driving memory control no longer dominates, recovery may be possible. Addressing issues relating to motivation presents a challenge to studying memory control in the laboratory, as we discuss next.

\section{The Role of Motivation in Motivated Forgetting}

Real situations that drive motivated forgetting have a critical ingredient that all laboratory research on memory control lacks: motivation. As discussed at the outset, people do not need special incentives to control awareness of unpleasant memories, as they are naturally motivated to not dwell on memories that make them angry, fearful, anxious, sad, or embarrassed.

When we study memory control in the laboratory, we are arguably studying a pale reflection of what must occur when people have an emotional incentive to succeed. Participants in most studies have no personal motive to suppress response words, apart from agreeing to cooperate with us. Absent a real personal motive for controlling awareness, we cannot know how effectively findings capture what happens in real cases. Indeed, when a true motive for suppressing is absent, other naturally occurring motivations will dominate. For instance, many participants are naturally motivated to appear smart, clever, or competent, and these motives very often drive them to intentionally rehearse suppression items when they know they are not supposed to (as discussed earlier) because they suspect they will be tested. Thus, unless special precautions are taken to ensure that people don't view the study as being about memory, the forces of motivation run counter to what the paradigm tries to achieve. We try to solve this problem of counter-motives by framing the task as being about the ability to ignore distracting things. We repeatedly stress that we 
are assessing this ability, which aligns subjects' desires to appear clever and competent with our task goals.

Nevertheless, research on motivated forgetting would benefit if participants had a personal motive for controlling memory, apart from compliance. There are two approaches to incorporating motivation into research on retrieval suppression. The first would be to study participants who, based of diagnostic criteria or other known facts, would have a motive to suppress certain content. For instance, participants with social phobia arguably have greater motivation to suppress awareness of stimuli with social content, and so might show larger negative control effects for that material. The second would be to experimentally induce a desire to suppress certain contents. Whether a creative way to induce motives could be devised that was still ethical remains to be seen.

\section{Integration with Research on Directed Forgetting}

Although retrieval suppression is an important model situation for understanding motivated forgetting, another body of work addresses related issues: directed forgetting. Research on directed forgetting examines whether people can intentionally forget recently encountered information. For example, in the item-method directed forgetting procedure (Bjork, 1972), people are presented stimuli (e.g., words, pictures) one at a time, and are told that following each item, they will receive instructions directing them to either remember or forget it. After the list is completed, a final recall or recognition test is given. The typical finding with the item method is that participants can recall or recognize substantially more remember items than forget items. In contrast, in the list-method directed forgetting procedure, a similar instruction is adopted, though a whole list is presented before the participants receive the remember or forget instruction, at which point a second list is presented. Here too, memory for the first list is impaired, compared to a first list that participants are asked to remember (Bjork et al., 1998; Geiselman et al., 1983, Johnson, 1994; see Golding \& MacLeod, 1998, for a review). In contrast, recognition memory for items forgotten with the list method is often intact, though not when recognition memory places greater demands on context memory (Sahakyan, Waldum, Benjamin, \& Bickett, 2009).

Both retrieval suppression and directed forgetting represent cases in which an effort to not think about an event or set of events leads to diminished recall of the unwanted memories. Despite this apparent similarity, however, the implementation of mnemonic control in these situations may vary. For instance, some have attributed item method directed forgetting to intentional truncation of encoding processes. By this view, until participants receive the cue to remember or forget, subjects intentionally halt elaborative encoding until they know what they are supposed to do, elaborating the item further only if it is to be remembered (e.g., Basden, Basden, \& Gargano, 1993). Others have argued for a role of cognitive control and response override in this procedure (Hourihan \& Taylor, 2006), a hypothesis supported by 
both behavioral (Fawcett \& Taylor, 2008) and imaging studies (Wylie, Fox, \& Taylor, 2008) that document the dependency of the forget instruction on attention and cognitive control systems. Regardless of which mechanism is at play, this task best captures situations in which we may prematurely terminate thoughts about an unpleasant experience to limit the footprint of that experience in our memories. The list method directed forgetting effect, by contrast, has been attributed to both to inhibition of the first list (Geiselman et al., 1983), and also to intentional shifts in mental context between the first list and the second (Sahakyan \& Kelley, 2002). In essence, list-method directed forgetting models the situation in which we try to get our mind off of something that has happened recently, by "changing gears." Retrieval suppression instead captures situations in which, encoding has already succeeded, and at some arbitrary point later a powerful reminder triggers an unwanted recollection. Given that the reminder cannot be escaped, mental context shift or truncated encoding are not viable options, and response override is likely to be more important. Thus, even though these tasks are superficially similar, memory control may be accomplished by different means and under different conditions.

An important goal of research on motivated forgetting is to understand the relationship between these different phenomena, the mechanisms they engage, and the situations they model. We would like to suggest the possibility that these tasks may all be viewed as engaging cognitive control, but perhaps targeted at different types of representations and processes. According to this flexible control hypothesis (Anderson, 2005), response override mechanisms may be flexibly targeted at different stages of memory, and at different processes. Item method directed forgetting may be a case of encoding suppression; list-method directed forgetting may reflect temporal context suppression; and negative control effects may reflect retrieval suppression, as we have discussed. This view is broadly compatible with the notion of response override as a general mechanism that can be targeted at different types of representations and processes. Alternative conceptualizations may be possible, however, and the important goal is to understand how these phenomena are related to one another.

\section{Integration with Research on Thought Suppression}

In apparent contradiction to the foregoing findings, a body of research on thought suppression has generally focused on the inefficacy of attempts to control thoughts. This research focuses on people's ability to suppress a single target thought over an extended period (usually $5 \mathrm{~min}$ ). In the typical "white bear" paradigm (Wegner, Schneider, Carter, \& White, 1987; see Wegner, 1994, Wenzlaff \& Wegner, 2000 for reviews), participants are told to spend 5 min suppressing all thoughts about a target thought (e.g., white bears) and to otherwise think about what they wish. If, however, they happen to think about white bears in the interim, they should ring a bell to indicate that the white bear intruded. After the 5 min period ends, they are given an additional $5 \mathrm{~min}$ "expression" period, in which they are told to think about 
white bears. Two general findings are sometimes observed. First, compared to an expression period, thought suppression instructions greatly reduce the frequency of the unwanted thought, though it rarely eliminates thoughts of the white bear completely. Second, a period of expression that follows a period of suppression often results in many more thoughts about white bears in comparison to a period of expression that does not follow suppression. The latter finding suggests that, ironically, attempting to suppress the unwanted thought causes a rebound in its accessibility, making it more accessible than it otherwise would have been. The conclusion usually reached in this literature is that thought suppression is counterproductive, and may lead to heightened levels of intrusive thoughts.

Here again, what might seem to be similar situations may not be served by the same mechanism. A key difference between thought suppression and the other methods is that the former makes explicit reference to a particular forbidden thought that is the very object of the task to be performed. The participants' understanding of the task is that their goal is to not think about white bears. As long as the participants try to accomplish that goal, it will be impossible to achieve it because simply remembering what they are supposed to do requires them to violate the goal. This contrasts with retrieval suppression, for example, in that the latter simply asks participants to prevent awareness of the memory that goes with a certain cue, without making reference to what that memory is. The fact that the goal of retrieval suppression task does not incorporate the very thing that is to be avoided may be a crucial feature that predicts when effective suppression is and is not possible. We propose this goal-integration theory as an account of this discrepancy between work on retrieval suppression and thought suppression. This hypothesis needs to be carefully examined to see if can help to disentangle when efforts at suppression will be productive. A careful analysis of the differing situations captured by these tasks, and the mechanisms involved will likely prove to be extremely helpful in relating research on mental control to clinical settings.

\section{Unconscious Influences of Suppressed Memories}

One final issue concerns whether retrieval suppression influences implicit access to traces. Many clinicians, particularly those from the psychoanalytic tradition subscribe to the view that even when memories cannot be retrieved, they continue to exert an influence on behavior and thought unconsciously. The possibility that such influences exist is intriguing. Yet, most of the work conducted thus far on retrieval suppression has focused on intentional, explicit retrieval of suppressed memories. Might memories that are intentionally suppressed continue to exert influence on people's behavior on indirect memory tests? Understanding whether and how such indirect influences might arise is theoretically important, and could also have profound implications for understanding the characteristics and consequences of retrieval suppression in clinical settings. 
At present, only one study has been conducted looking at implicit memory for recently suppressed materials (Kim \& Yi, 2008). Surprisingly, this study found that even indirect tests like perceptual identification of pictures show negative control effects, suggesting that suppression is not limited to conscious access to a trace. Clearly, however, further work needs to be done to assess the generality of this effect. One issue of particular interest is whether emotional learning associated with an unwanted memory might be preserved even when episodic memory for the experience is impaired. Thus, even when people cannot remember the negative event associated with a stimulus, they may experience emotional reactions to the stimulus that lead them to behave differently. Similarly, other indirect measures such as gaze pattern or other motor actions may reveal persisting influences. The discovery of intact influences of a prior experience, despite impaired memory would be informative at both a theoretical and clinical level.

\section{Beyond the Initial Act of Retrieval Suppression: How Memory Control Develops Over Time}

Motivated forgetting is unlikely to be accomplished in a single cognitive act or even in a short time, particularly for complex events with emotional content. Rather, it may require sustained effort, particularly if a person is confronted with reminders. For these reasons, motivated forgetting may best be viewed as an ongoing process supported by adapting mechanisms that limit awareness of the experience. Much of what is studied in the think/no-think paradigm, however, concerns the initial phases of memory control when one encounters reminders to a recently experienced event. Yet, the understanding of motivated forgetting likely requires an appreciation of how retrieval suppression accumulates over time, and how a person's coping response may adapt, neither of which are easily studied in controlled experiments. Here we discuss ways in which memory control may develop over time.

\section{The Intentionality Shift Theory}

After an unpleasant event, many people confront challenges in memory control, particularly if reminders are inescapable. The memories are recent and accessible. Given motivation to control awareness, however, intrusions diminish with time and effort. It is thus unavoidable that living with the demand to control an unwanted memory forces a person to improve with practice, as happens with all skills. This improvement will take the form of one or more habitual cognitive or affective responses to unwelcome reminders that suppress the experience and redirect thought. If practice continues over years, people may get very well adapted to the task. This protracted practice is a critical feature of real cases of motivated forgetting that is not easily studied in the laboratory. 
In our initial discussion of retrieval suppression, we suggested that memory control may shift from being intentional to unintentional, in part through a gradual shift in the approach people take (Anderson \& Green, 2001). According to this intentionality shift theory, people initially emphasize direct suppression because reminders elicit the unwanted memory involuntarily. Excluding the trace from awareness may often require direct suppression. Over time, however, people associate diversionary thoughts to the reminder, and may learn to retrieve those thoughts and pre-empt retrieval of the unwanted memory. Those thoughts may be other ideas about the reminder that a person selectively retrieves both as a means of occupying momentary awareness, and as a way of self-inflicting retrieval induced forgetting. Thus, extensive practice with unwelcome reminders may be associated with a progression from direct suppression to something more akin to our original selective retrieval hypothesis of motivated forgetting (Anderson, 2001; Bjork et al., 1998).

A gradual shift from a direct suppression approach to selective retrieval may ultimately permit people to forget not only the unpleasant experience, but also the process of suppressing it. There are two mechanisms by which this type of goal forgetting may occur. First, shifting from direct suppression to retrieving diversionary thoughts allows for a change in the goal people have from intentional control to retrieval of particular thoughts. Although the initial purpose of retrieving distracting thoughts is to intentionally suppress retrieval, this goal may be forgotten over time. If retrieval of thought substitutes reinstates inhibition of the unwanted event or further exaggerates interference, the shift from intentional suppression to selective retrieval should facilitate unawareness of the mental actions people take to avoid awareness of the unwanted memory. Second, as people become more practiced in retrieving diversionary thoughts in response to reminders, retrieval may become relatively automatic. If memories of earlier efforts to suppress are themselves associated to the reminder, this shift to retrieving alternative thoughts may ultimately suppress memories of control as well.

Although the intentionality shift theory is speculative, it may account for an important feature of recovered memories that may at first blush seem hard to reconcile with the emphasis we have placed on intentional retrieval suppression: the fact that people not only forget the original experience, but also how they came to forget it. This forgetting of the cognitions that one has about ones memories, including cognitions about intentional forgetting, might be termed metamemory amnesia, which we discuss next. A complete account of motivated forgetting thus requires an explanation both for how the memory itself was forgotten, and how the forgetting itself was forgotten.

\section{The Reinstatement Hypothesis}

The foregoing description assumes that forgetting becomes increasingly successful as people practice. Although this may be true for minor unpleasantness of life, 
more traumatic experiences may not progress as smoothly. Rather, truly upsetting experiences may be characterized by periodic resurgences in which the experience, not altogether forgotten, intrudes again, either in response to diminished capacity, new powerful reminders to the experience, or spontaneous recovery. These periodic challenges demand that retrieval suppression be reinstated. This may take the form of a return to intentional suppression or, instead, a resumption of diversionary thoughts.

Undoubtedly remindings of the unwanted experience are unpleasant, as are thoughts about the experience of being reminded. For these reasons, reinstatement of suppression will not merely be targeted at the original experience, but also thoughts that one has about it during the period of reminding. As such, even when a person remembers the experience for a period of time, they may not remember the remembering on later occasions. The reasons for this metamemory amnesia are straightforward. If we remember our thoughts -whether about perceptions or other thoughts - it is because these thoughts are stored in episodic memory as part of the content of experience. If a new trace is stored that encodes our thoughts about the memory, this new trace will share much in common with the original memory and be a natural target for retrieval suppression.

The reinstatement process thus predicts the phenomenon that Jonathan Schooler and colleagues called the "forgot it all along" effect, in which a person claims to have never recalled an experience when they have (Schooler, Bendiksen, \& Ambadar, 1997; Shobe \& Schooler, 2001). Schooler recounts cases of people who are convinced that they recovered a memory for childhood abuse never before retrieved, only to be corrected another person, who points out that the experience had been discussed years earlier. This forgot-it-all-along (FIA) effect has been modeled in the laboratory by the forgot-it-all-along paradigm (Arnold \& Lindsay, 2002; Geraerts, 2012, this volume; Geraerts, Arnold, Lindsay, Merckelbach, Jelicic, \& Hauer, 2006). Although forgetting prior remembering has been explained in terms of context dependent memory, real cases could just as easily be explained by reinstatement of retrieval suppression. Importantly, although some have taken the forgetting of prior remembering as evidence for an alternative hypothesis to motivated forgetting, the present analysis demonstrates that this conclusion is not demanded by this phenomenon. Rather, periodic recoveries and reinstatements are to be expected based on retrieval suppression, as is metamemory amnesia.

\section{The Influence of Other Forgetting Mechanisms Over Time}

Although we have emphasized retrieval suppression, it is not the only means of controlling unwanted memories. One can also truncate elaborative encoding, avoid retrieval cues where possible, and change physical context (Baddeley, Eysenck, \& Anderson, 2009). In real cases, a person will not rely exclusively on one mechanism, but will use any approach that succeeds. For instance, when a person intentionally stops a train of thought about an unpleasant experience, they are not only 
controlling their momentary affective state, but also limiting the encoding of elaborate traces that may pose fresh difficulties in memory control. By limiting encoding, a person reduces the integration of the unwanted thoughts with the rest of memory, increasing the potential for it to be forgotten (Anderson, 2001). This type of memory control is well modeled by item method directed forgetting, discussed earlier, which establishes that people can exert considerable influence over which experiences make it into memory.

When unpleasant experiences make it into memory, however, people will try to prevent retrieval from occurring. In this article, we have focused on cases in which reminders are inescapable and retrieval must be suppressed or redirected. The mechanisms engaged to control memory will likely be very different if reminders can be avoided altogether, however. Avoiding reminders eliminates the need to override retrieval or to retrain one's response to reminders. If retrieval suppression does not occur, then the consequences of retrieval suppression should also be avoided. Thus, avoiding reminders by changing physical contexts (e.g., moving to a different city or apartment) will probably work to reduce intrusions, but may not suppress the avoided memories if relevant cues do emerge. Hence, when people who have successfully avoided reminders in an initial context encounter reminders in a later and different context, they may experience full recollection of the unwanted memory (Brewin, 2012, this volume). Thus, cue avoidance and context shift deprive a person of a chance to retrain memory. This may be why abuse by a stranger more likely leads to continuous memory, whereas abuse by a parent is more likely to produce at least a partial forgetting of the abuse (Anderson, 2001; DePrince et al., 2012, this volume).

Truncated encoding and motivated context shifts may occur at different points in the development of a person's response to an unwanted memory. Truncated encoding may play a more important role early on, as a person strives to limit encoding and elaboration during or shortly after the experience. For instance, a person who tries to "remove themselves" psychologically from an unpleasant situation by focusing on entirely unrelated thoughts, or details of the physical environment is in effect is trying to redirect attention to other content to avoid encoding. Attempts to not dwell upon an event or think elaboratively about it afterwards serve a similar function. In contrast, for individuals who must live with inescapable reminders, motivated context shifts may occur later in the evolution of their response, when after a period of time such reminders are no longer present. For example, as a child matures into an adult, they will ultimately leave their home and perhaps move to a different city. Alternatively, the physical context may remain the same and a person may seek a shift in mental context by segmenting off whole periods of their past. When this type of context shift occurs, there is a qualitative change in the coping mechanisms: context shift makes retrieval suppression less relevant. As such, effects induced by retrieval suppression may subside (e.g., suppression will be released), although this change may go unnoticed, as reminders do not occur. This shift in physical (and likely emotional and mental) context sets the stage, potentially, for recovery of a forgotten memory, should the right cue appear. This process - the attempt to intentionally 
shift context to forget - is modeled by the list-method directed forgetting procedure of Robert and Elizabeth Bjork (Bjork, \& Bjork, 2003; Bjork, 1989; Geiselman et al., 1983) as discussed above.

More broadly, retrieval suppression is not synonymous with motivated forgetting. Motivated forgetting is achieved when people do not recall aspects of their past because they have engaged mechanisms to limit access to those experiences. The nature of those mechanisms may vary as long as they serve the broader motive of limiting awareness. Moreover, the motivated forgetting process is likely to be temporally extended, with the mechanisms engaged shaped by practice over time, and by changing circumstances of the individual controlling their memory. Thus, an understanding of how motivated forgetting emerges will require the development of laboratory models of different processes, and the incorporation of those into a broader framework of adaptive memory.

\section{Assessing the Role of Retrieval Suppression in Recovered Memories}

So far, we have focused on the mechanisms of retrieval suppression and how these mechanisms contribute to motivated forgetting. Our focus was not on the recovered memory debate, because the theoretical and practical themes of motivated forgetting transcend it. The purpose of this symposium, however, is to reconsider the scientific evidence in relation to this debate. Here we reflect on the implications of retrieval suppression for the recovered memory debate, and whether it may be one factor contributing to some cases of recovered memories.

Before beginning, it bears emphasis that this research cannot prove that any one recovered memory is real, and, if real, whether it may have been caused by suppression. The inconvenient truth is that even if a recovered memory is real, we will never know with certainty how and why it was forgotten, because the past events that led to the forgetting are unobservable. Because people reporting recovered memories often do not remember efforts to forget, evidence for retrieval suppression will often be indirect. As such, our comments should be taken as assessments of what may be possible in general, with conclusions about individual cases left to an assessment of its particulars. With those thoughts in mind, we divide our comments into what can and cannot reasonably be said.

\section{What Can Be Said}

The most basic implication of this research is that it provides an existence proof of a process that could, in principle, explain real cases of motivated forgetting, including cases of recovered memories. The work demonstrates that when people repeatedly 
confront reminders to an unwanted memory and take mental action to limit awareness of that memory, processes are engaged that achieve at least two basic outcomes: (a) they deprive a memory of the normal facilitation it would enjoy, and (b) they disrupt retention of the excluded trace, compared to when no reminders appear. Both of these actions, on average, reduce long-term accessibility of the suppressed trace, relative to other experiences of a similar age, which have the chance to be spontaneously retrieved given reminders. Reductions in accessibility are likely to be accomplished by several mechanisms, including direct suppression and thought substitution. Regardless of how these reductions are accomplished, however, one can certainly no longer say that there is no way, in principle, for motivated forgetting of abuse experiences to occur.

Of course, the present work was conducted with simple laboratory materials on very short time scales, and so proper caution must be exercised in generalizing these findings to events with considerably more complexity, emotional content, and personal relevance. Indeed, although these findings establish a process that could, in principle, produce these experiences, we emphasize that they do not, as yet, demonstrate a connection between recovered memories and retrieval suppression. As discussed in the preceding section, far more work needs to be done to develop retrieval suppression as a model of motivated forgetting. Thus, what has been established here is more properly viewed as a foundation for scientific development, rather than a completed proof of a process underlying motivated forgetting of abuse experiences.

Having said this, there is reason for optimism that the development of this case may succeed. Our retrieval suppression hypothesis was initially inspired by the higher incidence of self-reported forgetting for people abused by a parent than by a stranger. This pattern, on its face, is highly counter-intuitive, and led us to hypothesize that there may be something important about having to confront inescapable retrieval cues, coupled with a motivation to control awareness. Indeed, a similarly counter-intuitive retention pattern has been observed in the laboratory under the theoretically hypothesized conditions. The fact that these conditions in the laboratory are associated with enhanced forgetting lends credence to that hypothesis, and suggests that we may have identified one important contributor to some reports of recovered memories. Nevertheless, much work remains to connect this situation to the mechanisms studied in the laboratory. Indeed, for that connection to succeed, we must also fully explore why people are more likely to report forgetting abuse when it was committed by a caregiver.

Apart from providing an existence proof, the current work also provides a framework that suggests important hypotheses about when one is more likely to observe continuous versus discontinuous memory for abuse, and, moreover, the characteristics of forgetting under different circumstances. For instance, the current framework suggests that motivated forgetting accomplished by factors other than retrieval suppression, such as motivated context shifts, may differ in its characteristics from retrieval suppression. For instance, whereas reinstating the context of abuse may 
elicit strong recollections of the memory for people using motivated context shifts, it may be less effective as a means of eliciting retrieval of memories forgotten through retrieval suppression. If retrieval suppression has been maintained over the years in response to repeated reminders, the memory should be less accessible from those reminders and others.

\section{What Cannot Be Said}

Although it may be possible to develop a model of motivated forgetting built on the present work, we must clarify implications that do not, at present, follow from this work. First, as should be apparent from our discussion, we do not claim that all cases of memory recovery need to be produced by retrieval suppression. As we have emphasized, there are likely to be many cognitive routes to achieving reduced accessibility of unwanted memories, some of which will involve retrieval suppression, others of which will not. Retrieval suppression seems more likely to contribute in cases where a person is forced to confront unwelcome reminders over a long time, and is motivated to control awareness. As such, care should be taken to not overgeneralize the relationship of these findings to all cases of recovered memories.

Second, the present hypothesis frames motivated forgetting as a gradual process that people get better at with practice. Moreover, the process begins as an intentional act. For these reasons, the present mechanism does not address cases where memories are forgotten abruptly via an unconscious defense mechanism. So, for instance, if someone abruptly forgets a violent action shortly after it is taken, and has no recollection of the event, this does not obviously fall out of the processes envisioned here. Nevertheless, retrieval suppression might be involved, as some of the cases discussed here illustrate. Accounting for such cases requires one to provide additional arguments to why a process that normally develops with practice can be applied abruptly with dramatic effect, and with accompanying metamemory amnesia.

Third, the present framework does not imply that memories recovered after retrieval suppression will be accurate. The idea that suppressed memories may be preserved for many years and recovered in pristine form, seems highly implausible. Indeed, research on related inhibitory phenomena such as retrieval-induced forgetting, indicate that memories suppressed by inhibitory processes are actually more susceptible to distortion via misinformation effects than memories that have not been inhibited (see MacLeod \& Saunders, 2008, for a review). Thus, suppressing unwanted memories over a long time may fragment the experience and render it subject to distortion and reconstruction processes of the sort discussed in other contributions to this symposium. Thus, an understanding of the memorial consequences of motivated forgetting is likely to require consideration of retrieval suppression and distortion processes (Erdelyi, 2006). 


\section{Concluding Remarks}

A basic truth of human nature is that people don't like to feel bad. If there was ever a law of human behavior that could be counted on, it's that when someone is an aversive state, they will usually try to remove themselves from it. Similarly undeniable is the fact that not everything in memory is pleasant. Unlike unpleasant physical circumstances, however, one cannot as easily escape one's unpleasant memories. Wherever we go, they are with us. If people can be counted on to remove themselves from unpleasant states and if conscious awareness of some memories makes us feel unpleasant, it follows that people must be motivated to limit conscious awareness of certain memories. A scientific theory of forgetting cannot ignore the impact of these powerful motivational forces on shaping the fate of experience in long-term memory. What we remember and what we forget of our life experience is driven as much or more by our goals to regulate our current emotional state as it is by the passive, incidental factors traditionally emphasized in cognitive psychology.

The evidence that human beings try to control what they remember in service of regulating their emotional state is readily seen in the behavior of individuals and societies. As individuals, we alter our worlds to prevent being reminded of unpleasant experiences; we throw away objects given to us and we change apartments or towns; as societies, we even tear down buildings (e.g., the library associated with the Columbine shooting) or build new ones (e.g., the Millennium tower) to control what and how we remember. When forced to live with reminders, however, our only choice is to adjust our inner landscape. In this article, we discussed how this adjustment occurs. People control unwanted memories by engaging systems evolved to inhibit habitual responses to inhibit memories, making them harder to remember. The mechanisms that achieve this function are not exotic special-purpose responses to trauma, but rather are applications of broad mechanisms that achieve cognitive control. Thus, the tools to understand motivated forgetting are readily available in the armamentarium of cognitive neuroscience. Understanding how motivational forces alter what we remember of our lives provides key insights into what makes us resilient and shapes us as people.

Acknowledgments Preparation of this article was supported by National Science Foundation grant 0643321. The authors would like to thank Robert Bjork, Steve Smith, Karl-Heinz Bauml, Lili Sahakyan, Tracy Taylor-Hemick, Paula Hertel, Jutta Joormann, Kepa Paz-Alanso, Roland Benoit, and Zara Bergstrom for useful comments on this manuscript.

\section{Appendix A}

The 32 published articles on which the meta-analysis in Fig. 3 (right panel) is based. Note that four published articles are not included either because they used different dependent measures or did not fully report recall data (see caption, Fig. 3). 


\begin{tabular}{|c|c|c|}
\hline Reference & Participants & Suppressed Material \\
\hline Anderson, M. C. \& Green, C., 2001 & normals $(n=96)$ & neutral words \\
\hline Hertel, P. T. \& Gerstle, M., 2003 & controls $(n=32)$ and dysphorics $(n=32)$ & positive and negative words \\
\hline Anderson, M. C. et al., 2004 & normals $(n=24)$ & neutral words \\
\hline Hertel, P. T. \& Calcaterra, G., 2005 & normals $(n=72)$ & neutral words \\
\hline Joormann, J. et al., 2005 & controls $(n=24)$ and depressed $(n=32)$ & neutral words \\
\hline Wessel, I. et al., 2005 & low $(n=33)$ and high $(n=35)$ dissociators & neutral words \\
\hline Bulevich, J. B. et al., 2006 & normals $(n=66)$ & neutral words \\
\hline Bergstrom, Z. M. et al., 2007 & normals $(n=31)$ & neutral words \\
\hline Depue, B. E. et al., 2007 & normals $(n=16)$ & negative IAPS scenes \\
\hline Kim, K. et al., 2007 & repressors $(n=29)$ and nonrepressors $(n=46)$ & neutral and negative words \\
\hline Lee, Y. et al., 2007 & normals $(n=82)$ & neutral words \\
\hline Salame, P. \& Danion, J., 2007 & schizophrenics $(n=23)$ and controls $(n=24)$ & neutral words \\
\hline Hertel, P. T. \& Mahan, A., 2008 & dysphoric $(n=18)$ and nondysphoric $(n=18)$ & words related and unrelated to cue \\
\hline Bergstrom, Z. M. et al., 2009 & normals $(n=48)$ & neutral words \\
\hline Hanslmayr, S. et al., 2009 & normals $(n=24)$ & neutral words \\
\hline Hotta, C. \& Kawaguchi, J., 2009 & normals $(n=24)$ & neutral words \\
\hline Joormann, J. et al., 2009 & controls $(n=45)$ and MDDs $(n=45)$ & negative words \\
\hline Mecklinger, A. et al., 2009 & normals $(n=24)$ & neutral words \\
\hline Paz-Alonso, P. M. et al., 2009 & $8-12$ year olds $(n=40)$ and normals $(n=30)$ & neutral words \\
\hline Tomlinson, T. D. et al., 2009 & normals $(n=84)$ & neutral words \\
\hline Tramoni, E. et al., 2009 & functional amnesia patient $(n=1)$ and controls $(n=12)$ & neutral words \\
\hline Dieler, A. C. et al., 2010 & normals $(n=16)$ & positive, negative and neutral words \\
\hline Hanslmayr, S. et al., 2010 & normals $(n=48)$ & neutral words \\
\hline Hertel, P. \& McDaniel, L., 2010 & repressors $(n=36)$ and nonrepressors $(n=36)$ & negative words \\
\hline Lambert, A. J. et al., 2010 & normals $(n=126)$ & neutral words \\
\hline Lemoult, J. et al., 2010 & normals $(n=56)$ & neutral words \\
\hline Norby, S. et al., 2010 & normals $(n=48)$ & neutral and negative words \\
\hline Wessel, I. et al., 2010 & morning and evening types $(n=80)$ & neutral words \\
\hline Anderson, M. C. et al., 2011 & older adults ( $n=61$ ) and younger adults ( $n=62)$ & neutral words \\
\hline Meier, B. et al., 2011 & normals $(n=20)$ & neutral words \\
\hline Murray, B. D. et al., 2011 & older adults ( $n=79)$ and younger adults $(n=87)$ & neutral words \\
\hline Waldhauser, G. T. et al., 2011 & high anxiety and low anxiety (total $n=41$ ) & neutral words \\
\hline
\end{tabular}

\section{References}

Adams, Z. W., Derefinko, K. J., Milich, R., \& Fillmore, M. T. (2008). Inhibitory functioning across ADHD subtypes: Recent findings, clinical implications, and future directions. Developmental Disabilities Research Reviews, 14(4), 268-275. doi:10.1002/ddrr.37.

Allen, G. A., Mahler, W. A., \& Estes, W. K. (1969). Effects of recall tests on long-term retention of paired associates. Journal of Verbal Learning and Verbal Behavior, 8(4), 463-470.

Anderson, M. C. (2001). Active forgetting: Evidence for functional inhibition as a source of memory failure. Journal of Aggression, Maltreatment, and Trauma, 4(2), 185-210.

Anderson, M. C. (2003). Rethinking interference theory: Executive control and the mechanisms of forgetting. Journal of Memory and Language, 49, 415-445.

Anderson, M. C. (2005). The role of inhibitory control in forgetting unwanted memories: A consideration of three methods. In C. MacLeod \& B. Uttl (Eds.), Dynamic cognitive processes (pp. 159-190). Tokyo: Springer. 
Anderson, M. C., Bjork, R., \& Bjork, E. (1994). Remembering can cause forgetting: Retrieval dynamics in long-term memory. Journal of Experimental Psychology: Learning, Memory, and Cognition, 20(5), 1063-1087.

Anderson, M. C., \& Green, C. (2001). Suppressing unwanted memories by executive control. Nature, 410, 366-369.

Anderson, M. C., Reinholz, J., Kuhl, B., \& Mayr, U. (2011). Intentional suppression of unwanted memories grows more difficult as we age. Psychology and Aging, 26, 397-405.

Anderson, M. C., \& Levy, B. J. (2006). Encouraging the nascent cognitive neuroscience of repression. Behavioral and Brain Sciences, 29(5), 511-513.

Anderson, M. C., Ochsner, K. N., Kuhl, B., Cooper, J., Robertson, E., Gabrieli, S. W., et al. (2004). Neural systems underlying the suppression of unwanted memories. Science, 303(5655), 232-235. doi:10.1126/science.1089504.

Anderson, M. C., \& Spellman, B. A. (1995). On the status of inhibitory mechanisms in cognition: Memory retrieval as a model case. Psychological Review, 102(1), 68-100.

Anderson, M. C., \& Weaver, C. (2009). Inhibitory control over action and memory. In L. R. Squire (Ed.), The new encyclopedia of neuroscience (pp. 153-163). Oxford: Elsevier Ltd. doi:10.1016/ B978-008045046- 9.00421-6.

Arnold, M. M., \& Lindsay, D. S. (2002). Remembering remembering. Journal of Experimental Psychology: Learning, Memory, and Cognition, 28, 521-529.

Aron, A. R., Fletcher, P. C., Bullmore, E. T., Sahakian, B. J., \& Robbins, T. W. (2003). Stop-signal inhibition disrupted by damage to right inferior frontal gyrus in humans. Nature Neuroscience, 6(2), 115-116. doi:10.1038/nn1003.

Aslan, A., \& Bäuml, K. T. (2010). Retrieval-induced forgetting in young children. Psychonomic Bulletin and Review, 17(5), 704-709. doi:10.3758/PBR.17.5.704.

Baddeley, A. D., Eysenck, M., \& Anderson, M. C. (2009). Memory. Hove: Psychology Press.

Band, G. P., \& van Boxtel, G. J. (1999). Inhibitory motor control in stop paradigms: Review and reinterpretation of neural mechanisms. Acta Psychologica, 101(2-3), 179-211.

Barkley, R. (1997). Behavioral inhibition, sustained attention, and executive functions: Constructing a unifying theory of ADHD. Psychological Bulletin, 121(1), 65-94.

Basden, B. H., Basden, D. R., \& Gargano, G. J. (1993). Directed forgetting in implicit and explicit memory tests: A comparison of methods. Journal of Experimental Psychology: Learning, Memory, and Cognition, 19(3), 603-616.

Baumeister, R. (2003). Ego depletion and self-regulation failure: A resource model of self-control. Alcoholism, Clinical and Experimental Research, 27(2), 281-284. doi:10.1097/01. ALC.0000060879.61384.A4.

Baumeister, R., Bratslavsky, E., Muraven, M., \& Tice, D. M. (1998). Ego depletion: Is the active self a limited resource. Journal of Personality and Social Psychology, 74(5), 1252-1265.

Bekker, E. M., Kenemans, J. L., \& Verbaten, M. N. (2005). Source analysis of the N2 in a cued Go/ NoGo task. Cognitive Brain Research, 22(2), 221-231. doi:10.1016/j.cogbrainres.2004.08.011.

Benjamin, A. (2010). In Benjamin Aaron (Ed.), Successful remembering and successful forgetting: A festschrift in honor of Robert A. Bjork. New York: Psychology Press.

Bergstrom, Z. M., Anderson, M. C., Buda, M., Simons, J., \& Richardson-Klavehn, A. (submitted). Intentional retrieval suppression can conceal guilty knowledge in ERP memory detection tests.

Bergström, Z. M., de Fockert, J. W., \& Richardson-Klavehn, A. (2009). ERP and behavioural evidence for direct suppression of unwanted memories. NeuroImage, 48(4), 726-737. doi:10.1016/j.neuroimage.2009.06.051.

Bergström, Z. M., Velmans, M., de Fockert, J., \& Richardson-Klavehn, A. (2007). ERP evidence for successful voluntary avoidance of conscious recollection. Brain Research, 1151, 119-133. doi:10.1016/j.brainres.2007.03.014.

Bjork, R. A. (1972). Theoretical implications of directed forgetting. In A. W. Melton \& E. Martin (Eds.), Coding processes in human memory (pp. 217-235). Washington, DC: Winston.

Bjork, R. A. (1975). Retrieval as a memory modifier. In R. Solso (Ed.), Information processing and cognition: The Loyola Symposium (pp. 123-144). Hillsdale, NJ: Lawrence Erlbaum Associates. 
Bjork, R. A. (1989). Retrieval inhibition as an adaptive mechanism in human memory. In H. L. Roediger \& F. I. M. Craik (Eds.), Varieties of memory and consciousness: Essays in honour of Endel Tulving (pp. 309-330). Hillsdale, NJ: Erlbaum.

Bjork, E. L., \& Bjork, R. A. (2003). Intentional forgetting can increase, not decrease, the residual influences of to-be-forgotten information. Journal of Experimental Psychology: Learning, Memory, and Cognition, 29, 524-531.

Bjork, E. L., Bjork, R. A., \& Anderson, M. C. (1998). Varieties of goal-directed forgetting. In J. M. Golding \& C. M. MacLeod (Eds.), Intentional forgetting: Interdisciplinary approaches (pp. 103-137). Hillsdale, NJ: Erlbaum.

Bokura, H., Yamaguchi, S., \& Kobayashi, S. (2001). Electrophysiological correlates for response inhibition in a Go/NoGo task. Clinical Neurophysiology, 112(12), 2224-2232.

Booth, J. R., Burman, D. D., Meyer, J. R., Lei, Z., Trommer, B. L., Davenport, N. D., et al. (2005). Larger deficits in brain networks for response inhibition than for visual selective attention in attention deficit hyperactivity disorder (ADHD). The Journal of Child Psychology and Psychiatry, 46(1), 94-111. doi:10.1111/j.1469-7610.2004.00337.x.

Brewin, C. R. (2012, this volume). A theoretical framework for understanding recovered memory experiences. In R. F. Belli (Ed.), True and false recovered memories: Toward a reconciliation of the debate (pp. 149-173). Vol. 58: Nebraska Symposium on Motivation. New York: Springer.

Brown, A. S. (1976). Spontaneous recovery and human learning. Psychological Bulletin, 83, 321-338.

Bulevich, J. B., Roediger, H. L., Balota, D. A., \& Butler, A. C. (2006). Failures to find suppression of episodic memories in the think/no-think paradigm. Memory \& Cognition, 34(8), 1569-1577.

Butler, A. J., \& James, K. H. (2010). The neural correlates of attempting to suppress negative versus neutral memories. Cognitive, Affective, \& Behavioral Neuroscience, 10(2), 182-194. doi:10.3758/CABN.10.2.182.

Cameron, C. (1993, April). Recovering memories of childhood sexual abuse: A longitudinal report. Paper presented at the Western Psychological Association convention, Phoenix, AZ, USA.

Carrier, M., \& Pashler, H. (1992). The influence of retrieval on retention. Memory \& Cognition, 20(6), 633-642.

Casey, B. J., Castellanos, F. X., Giedd, J. N., Marsh, W. L., Hamburger, S. D., Schubert, A. B., et al. (1997). Implication of right frontostriatal circuitry in response inhibition and attentiondeficit/hyperactivity disorder. Journal of the American Academy of Child and Adolescent Psychiatry, 36(3), 374-383. doi:10.1097/00004583-199703000-00016.

Chan, J. C. K. (2009). Long-term effects of testing on the recall of nontested materials. Memory, 18(1), 49-57. doi:10.1080/09658210903405737.

Conroy, R., \& Salmon, K. (2005). Selective postevent review and childrens' memory for nonreviewed materials. Journal of Experimental Child Psychology, 90(4), 185-207. doi:10.1016/j. jecp. 2004.11.004.

Conroy, R., \& Salmon, K. (2006). Talking about parts of a past experience: The impact of discussion style and event structure on memory for discussed and nondiscussed information. Journal of Experimental Child Psychology, 95(4), 278-297. doi:10.1016/j.jecp. 2006.06.001.

DePrince, A., Brown, L., Cheit, R., Freyd, J., Gold, S. N., Pezdek, K., \& Quina, K. (2012, this volume). Motivated forgetting and misremembering: Perspectives from betrayal trauma theory. In R. F. Belli (Ed.), True and false recovered memories: Toward a reconciliation of the debate (pp. 193-242). Vol. 58: Nebraska Symposium on Motivation. New York: Springer.

Depue, B. E., Banich, M. T., \& Curran, T. (2006). Suppression of emotional and nonemotional content in memory: Effects of repetition on cognitive control. Psychological Science, 17(5), 441-447. doi:10.1111/j.1467-9280.2006.01725.x.

Depue, B. E., Burgess, G. C., Willcutt, E. G., Bidwell, L. C., Ruzic, L., \& Banich, M. T. (2010). Symptom-correlated brain regions in young adults with combined-type ADHD: Their organization, variability, and relation to behavioral performance. Psychiatry Research: Neuroimaging, 182(2), 96-102. doi:10.1016/j.pscychresns.2009.11.011.

Depue, B. E., Burgess, G. C., Willcutt, E. G., Ruzic, L., \& Banich, M. T. (2010). Inhibitory control of memory retrieval and motor processing associated with the right lateral prefrontal cortex: 
Evidence from deficits in individuals with ADHD. Neuropsychologia, 48(13), 3909-3917. doi:10.1016/j.neuropsychologia.2010.09.013.

Depue, B. E., Curran, T., \& Banich, M. T. (2007). Prefrontal regions orchestrate suppression of emotional memories via a two-phase process. Science, 317(5835), 215-219. doi:10.1126/ science.1139560.

Detre, G. J., Natarajan, A., \& Norman, K. A. (2010, November). Moderate memory activation leads to forgetting in the Think-No Think paradigm. Poster presented at the Annual Meeting of the Society for Neuroscience, San Diego, CA, USA.

Dieler, A. C., Plichta, M. M., Dresler, T., \& Fallgatter, A. J. (2010). Suppression of emotional words in the Think/No-Think paradigm investigated with functional near-infrared spectroscopy. International Journal of Psychophysiology, 78(2), 129-135. doi:10.1016/j.ijpsycho.2010.06.358.

Donkers, F., \& van Boxtel, G. J. M. (2004). The N2 in go/no-go tasks reflects conflict monitoring not response inhibition. Brain and Cognition, 56, 165-176.

Duzel, E., Cabeza, R., Picton, T. W., Yonelinas, A. P., Scheich, H., Heinze, H. J., et al. (1999). Task-related and item-related brain processes of memory retrieval. Proceedings of the National Academy of Sciences, 96, 1794-1799.

Eimer, M. (1993). Effects of attention and stimulus probability on ERPs in a Go/Nogo task. Biological Psychology, 35(2), 123-138.

Eldridge, L. L., Knowlton, B. J., Furmanski, C. S., Bookheimer, S. Y., \& Engel, S. A. (2000). Remembering episodes: A selective role for the hippocampus during retrieval. Nature Neuroscience, 3, 1149.

Erdelyi, M. H. (1996). The recovery of unconscious memories: Hypermnesia and reminiscence. Chicago: The University of Chicago Press.

Erdelyi, M. H. (2006). The unified theory of repression. Behavioral and Brain Sciences, 29(5), 499-551. doi:10.1017/S0140525X06009113.

Erdelyi, M., \& Kleinbard, J. (1978). Has Ebbinghaus decayed with time? The growth of recall (hypermnesia) over days. Journal of Experimental Psychology: Human Learning and Memory, 4(4), 275-289.

Falkenstein, M. (2006). Inhibition, conflict and the Nogo-N2. Clinical Neurophysiology, 117(8), 1638-1640. doi:10.1016/j.clinph.2006.05.002.

Falkenstein, M., Hoormann, J., \& Hohnsbein, J. (1999). ERP components in Go/Nogo tasks and their relation to inhibition. Acta Psychologica, 101(2-3), 267-291.

Fawcett, J. M., \& Taylor, T. L. (2008). Forgetting is effortful: Evidence from reaction time probes in an item-method directed forgetting task. Memory \& Cognition, 36(6), 1168-1181.

Feldman-Summers, S., \& Pope, K. S. (1994). The experience of 'forgetting' child abuse: A national survey of psychologists. Journal of Consulting and Clinical Psychology, 3, 626-639.

Ford, R. M., Keating, S., \& Patel, R. (2004). Retrieval-induced foregetting: A developmental study. Developmental Psychology, 22(4), 5850-603.

Freyd, J. J. (1996). Betrayal trauma: The logic of forgetting childhood abuse. Cambridge, MA: Harvard University Press.

Freyd, J. J., DePrince, A. P., \& Gleaves, D. (2007). The state of betrayal trauma theory: Reply to McNally - conceptual issues and future directions. Memory, 15, 295-311.

Freyd, J. J., Deprince, A. P., \& Zurbriggen, E. L. (2006). Self-reported memory for abuse depends upon victim-perpetrator relationship. Journal of Trauma \& Dissociation, 2(3), 5-15.

Friedman, D., \& Johnson, R. (2000). Event-related potential (ERP) studies of memory encoding and retrieval: A selective review. Microscopy Research and Technique, 51(1), 6-28. doi:10.1002/1097-0029(20001001)51:1<6::AID-JEMT2>3.0.CO;2-R.

Garavan, H., Ross, T. J., Murphy, K., Roche, R. A. P., \& Stein, E. A. (2002). Dissociable executive functions in the dynamic control of behavior: Inhibition, error detection, and correction. NeuroImage, 17(4), 1820-1829.

Garcia-Bajos, E., Migueles, M., \& Anderson, M. C. (2009). Script knowledge modulates retrieval-induced forgetting for eyewitness events. Memory, 17(1), 92-103. doi:10.1080/ 09658210802572454. 
Geiselman, R. E., Bjork, R. A., \& Fishman, D. L. (1983). Disrupted retrieval in directed forgetting: A link with posthypnotic amnesia. Journal of Experimental Psychology: General, 112(1), 58-72.

Geraerts, E., Arnold, M. M., Lindsay, D. S., Merckelbach, H., Jelicic, M., \& Hauer, B. (2006). Forgetting of prior remembering in people reporting recovered memories of childhood sexual abuse. Psychological Science, 17, 1002-1008.

Geraerts, E. (2012, this volume). Cognitive underpinnings of recovered memories of childhood abuse. In R. F. Belli (Ed.), True and false recovered memories: Toward a reconciliation of the debate (pp. 175-191). Vol. 58: Nebraska Symposium on Motivation. New York: Springer.

Geraerts, E., McNally, R. J., Jelicic, M., Merckelbach, H., \& Raymaekers, L. (2008). Linking thought suppression and recovered memories of childhood sexual abuse. Memory, 16(1), 22-28. doi:10.1080/09658210701390628.

Goernert, P. (2005). Source-monitoring accuracy across repeated tests following directed forgetting. British Journal of Psychology, 96(2), 231-247.

Goernert, P. N., \& Wolfe, T. (1997). Is there hypermnesia and reminiscence for information intentionally forgotten? Canadian Journal of Experimental Psychology, 51(3), 231-240.

Goldberg, L. R., \& Freyd, J. J. (2006). Self-reports of potentially traumatic experiences in an adult community sample: Gender differences and test-retest stabilities of the items in a brief betrayaltrauma survey. Journal of Trauma \& Dissociation, 7(3), 39-63.

Golding, J. M., \& MacLeod, C. M. (1998). Intentional forgetting: Interdisciplinary approaches. Mahwah, NJ: Erlbaum.

Goodman, G. S., Ghetti, S., Quas, J. A., Edelstein, R. S., Alexander, K. W., Redlich, A. D., et al. (2003). A prospective study of memory for child sexual abuse: New findings relevant to the repressed-memory controversy. Psychological Science, 14, 113-118.

Goodmon, L. B., \& Anderson, M. C. (2011). Semantic integration as a boundary condition on inhibitory processes in episodic retrieval. Journal of Experimental Psychology: Learning, Memory, and Cognition, 37(2), 416-436. doi:10.1037/a0021963.

Hagger, M. S., Wood, C., Stiff, C., \& Chatzisarantis, N. L. D. (2010). Ego depletion and the strength model of self-control: A meta-analysis. Psychological Bulletin, 136(4), 495-525. doi:10.1037/a0019486.

Hanslmayr, S., Leipold, P., \& Bauml, K. (2010). Anticipation boosts forgetting of voluntarily suppressed memories. Memory, 18, 252-257. doi:10.1080/09658210903476548.

Hanslmayr, S., Leipold, P., Pastötter, B., \& Bäuml, K. (2009). Anticipatory signatures of voluntary memory suppression. The Journal of Neuroscience, 29(9), 2742-2747. doi:10.1523/JNEUROSCI. 4703-08.2009.

Harnishfeger, K. K., \& Pope, R. S. (1996). Intending to forget: The development of cognitive inhibition in directed forgetting. Journal of Experimental Child Psychology, 62(2), 292-315. doi:10.1006/jecp. 1996.0032.

Hasher, L., \& Zacks, R. T. (1988). Working memory, comprehension, and aging: A review and a new view. In H. Bower (Ed.), The psychology of learning and motivation (Vol. 22, pp. 193225). San Diego, CA: Academic.

Henkel, L. A. (2004). Erroneous memories arising from repeated attempts to remember. Journal of Memory and Language, 50, 26-46.

Henkel, L., \& Koffman, K. J. (2004). Memory distortions in coerced false confessions: A source monitoring framework analysis. Applied Cognitive Psychology, 18(5), 567-588.

Hertel, P. T. (1994). Depressive deficits in memory: Implications of research and theory for memory improvement following traumatic brain injury. NeuroRehabilitation, 4, 143-150.

Hertel, P. T. (1998). Relation between rumination and impaired memory in dysphoric moods. Journal of Abnormal Psychology, 107(1), 166-172.

Hertel, P. T., Large, D., Dahl, E., \& Levy, A. (2011). Suppression-induced forgetting on a freeassociation test.

Hertel, P. T., \& Calcaterra, G. (2005). Intentional forgetting benefits from thought substitution. Psychonomic Bulletin and Review, 12(3), 484-489. 
Hertel, P. T., \& Gerstle, M. (2003). Depressive deficits in forgetting. Psychological Science, 14(6), 573-578.

Hertel, P., \& Mahan, A. (2008). Depression-related differences in learning and forgetting responses to unrelated cues. Acta Psychologica, 127(3), 636-644. doi:10.1016/j.actpsy.2007.11.004.

Hertel, P., \& McDaniel, L. (2010). The suppressive power of positive thinking: Aiding suppressioninduced forgetting in repressive coping. Cognition and Emotion, 24(7), 1239-1249.

Hotta, C., \& Kawaguchi, J. (2009). Self-initiated use of thought substitution can lead to long term forgetting. Psychologia, 52(1), 41-49.

Hourihan, K. L., \& Taylor, T. L. (2006). Cease remembering: Control processes in directed forgetting. Journal Of Experimental Psychology: Human Perception and Performance, 32(6), 1354 1365. doi:10.1037/0096-1523.32.6.1354.

Huddleston, E., \& Anderson, M.C. (in preparation). Retrieval suppression modulates activation in content-specific neocortical areas.

Hulbert, J. C., Anderson, M. C., \& Kuhl, B. (in preparation). Enhanced inhibitory control over memory in people with extensive traumatic experience.

Hulbert, M. C., Shivde, G. S., \& Anderson, M. C. (2011). Evidence against associative blocking as a cause of cue-independent retrieval-induced forgetting. Experimental Psychology.

Iversen, S., \& Mishkin, M. (1970). Perseverative interference in monkeys following selective lesions of inferior prefrontal convexity. Experimental Brain Research, 11(4), 376-386.

Johnson, M. K. (1994). Binding complex memories: The role of reactivation and the hippocampus. In D. L. Schacter \& E. Tulving (Eds.), Memory systems (pp. 311-350). Cambridge, MA: The MIT Press.

Johnson, R., Kreiter, K., Russo, B., \& Zhu, J. (1998). A spatio-temporal analysis of recognitionrelated event-related brain potentials. International Journal of Psychophysiology, 29(1), 83-104.

Joormann, J., Hertel, P. T., Brozovich, F., \& Gotlib, I. H. (2005). Remembering the good, forgetting the bad: Intentional forgetting of emotional material in depression. Journal of Abnormal Psychology, 114(4), 640-648. doi:10.1037/0021-843X.114.4.640.

Joormann, J., Hertel, P. T., Lemoult, J., \& Gotlib, I. H. (2009). Training forgetting of negative material in depression. Journal of Abnormal Psychology, 118(1), 34-43. doi:10.1037/a0013794.

Joormann, J., Yoon, K. L., \& Zetsche, U. (2007). Cognitive inhibition in depression. Applied and Preventive Psychology, 12, 128-139.

Karpicke, J. D., \& Roediger, H. L. (2008). The critical importance of retrieval for learning. Science, 319(5865), 966-968. doi:10.1126/science.1152408.

Kikuchi, H., Fujii, T., Abe, N., Suzuki, M., Takagi, M., Mugikura, S., et al. (2010). Memory repression: Brain mechanisms underlying dissociative amnesia. Journal of Cognitive Neuroscience, 22(3), 602-613. doi:10.1162/jocn.2009.21212.

Kim, K., \& Yi, D. (2008, November). Perceptual consequences of memory suppression. Poster presented at the Annual Meeting of the Society for Neuroscience, Washington, DC, USA.

Kim, K., Yi, D., Yang, E., \& Lee, K. (2007). What makes repressors good suppressors? The effect of trait anxiety. Korean Journal of Psychology, 26, 261-277.

Kopp, B., Mattler, U., Goertz, R., \& Rist, F. (1996). N2, P3 and the lateralized readiness potential in a nogo task involving selective response priming. Electroencephalography and Clinical Neurophysiology, 99(1), 19-27.

Lambert, A. J., Good, K. S., \& Kirk, I. J. (2010). Testing the repression hypothesis: Effects of emotional valence on memory suppression in the think - no think task. Consciousness and Cognition, 19(1), 281-293. doi:10.1016/j.concog.2009.09.004.

Landauer, T. K., \& Bjork, R. A. (1978). Optimum rehearsal patterns and name learning. In M. M. Gruneberg, P. E. Morris, \& R. N. Sykes (Eds.), Practical aspects of memory (pp. 625-632). New York: Academic.

Lavric, A., Pizzagalli, D. A., \& Forstmeier, S. (2004). When 'go' and 'nogo' are equally frequent: ERP components and cortical tomography. The European Journal of Neuroscience, 20(9), 2483-2488. doi:10.1111/j.1460-9568.2004.03683.x. 
Lee, Y., Lee, H., \& Tsai, S. (2007). Effects of post-cue interval on intentional forgetting. British Journal of Psychology, 98(2), 257-272. doi:10.1348/000712606X120410.

LeMoult, J., Hertel, P. T., \& Joorman, J. (2010). Training the forgetting of negative words: The role of direct suppression and the relation to stress reactivity. Applied Cognitive Psychology, 24, 365-375.

Levy, B. J., \& Anderson, M. C. (2002). Inhibitory processes and the control of memory retrieval. Trends in Cognitive Sciences, 6(7), 299-305.

Levy, B. J., \& Anderson, M. C. (2008). Individual differences in the suppression of unwanted memories: The executive deficit hypothesis. Acta Psychologica, 127(3), 623-635. doi:10.1016/j. actpsy.2007.12.004.

Levy, B. J., \& Wagner, A. D. (2011). Cognitive control and right ventrolateral prefrontal cortex: reflexive reorienting, motor inhibition, and action updating. Annals of the New York Academy of Sciences, 1224, 40-62.

Logan, G. D., Cowan, W., \& Davis, K. (1994). On the ability to inhibit thought and action: A users' guide to the stop-signal paradigm. In D. D. Carr \& T. H. Carr (Eds.), Inhibitory processes in attention, memory, and language (pp. 189-239). San Diego, CA: Academic.

Luria, A. R. (1966). Higher cortical functions in man. New York: Basic Books.

Lustig, C., Hasher, L., \& Tonev, S. T. (2001a). Inhibitory control over the present and the past. European Journal of Cognitive Psychology, 13, 107-122.

MacDonald, A. W., Cohen, J. D., Stenger, V. A., \& Carter, C. S. (2000). Dissociating the role of the dorsolateral prefrontal and anterior cingulate cortex in cognitive control. Science, 288(5472), $1835-1838$.

MacLeod, M., \& Macrae, C. (2001). Gone but not forgotten: The transient nature of retrievalinduced forgetting. Psychological Science, 12(2), 148-152.

MacLeod, M. D., \& Saunders, J. (2008). Retrieval inhibition and memory distortion: Negative consequences of an adaptive process. Current Directions in Psychological Science, 17(1), 26-30.

Marx, B. P., Marshall, P. J., \& Castro, F. (2008). The moderating effects of stimulus valence and arousal on memory suppression. Emotion, 8(2), 199-207. doi:10.1037/1528-3542.8.2.199.

McNally, R. J. (2007). Betrayal trauma theory: A critical appraisal. Memory, 15, 280-294.

Mecklinger, A., Parra, M., \& Waldhauser, G. T. (2009). ERP correlates of intentional forgetting. Brain Research, 1255(C), 132-147. doi:doi:10.1016/j.brainres.2008.11.073.

Meier, B., König, A., Parak, S., \& Henke, K. (2011). Suppressed, but not forgotten. Swiss Journal of Psychology, 70(1), 5-11. doi:10.1024/1421-0185/a000033.

Menon, V., Adleman, N. E., White, C. D., Glover, G. H., \& Reiss, A. L. (2001). Error-related brain activation during a Go/NoGo response inhibition task. Human Brain Mapping, 12(3), 131-143.

Murray, B. D., Muscatell, K. A., \& Kensinger, E. A. (2011). Effects of Emotion and Age on Performance During a Think/No-Think Memory Task Psychology and Aging in press.

Nigg, J. (2000). On inhibition/disinhibition in developmental psychopathology: Views from cognitive and personality psychology and a working inhibition taxonomy. Psychological Bulletin, 126(2), 220-246.

Nigg, J. (2001). Is ADHD a disinhibitory disorder? Psychological Bulletin, 127(5), 571-598. doi:10.1037//0033-2909.127.5.571.

Nørby, S., Lange, M., \& Larsen, A. (2010). Forgetting to forget: On the duration of voluntary suppression of neutral and emotional memories. Acta Psychologica, 133(1), 73-80. doi:10.1016/j. actpsy.2009.10.002.

Norman, W., \& Shallice, T. (1986). Attention to action. In R. J. Davidson, G. E. Schwartz, \& D. Shapiro (Eds.), Consciousness and self regulation: Advances in research and theory (Vol. 4, pp. 1-18). New York: Plenum.

Ogle, C. M., \& Paz-Alonso, P. M. (in preparation). Developmental changes in the suppression of emotional memories.

Oosterlaan, J., Logan, G. D., \& Sergeant, J. A. (1998). Response inhibition in AD/HD, CD, comorbid $\mathrm{AD} / \mathrm{HD}+\mathrm{CD}$, anxious, and control children: A meta-analysis of studies with the stop task. Journal of Child Psychology and Psychiatry, 39(3), 411-425.

Pavlov, I. P. (1927) Conditioned reflexes (G. V. Anrep, Trans.). London: Oxford University Press. 
Payne, D. G. (1987). Hypermnesia and reminiscence in recall: A historical and empirical review. Psychological Bulletin, 101(1), 5-27. doi:10.1037/0033-2909.101.1.5.

Paz-Alonso, P. M., Ghetti, S., Wendelken, C., Anderson, M. C., \& Bunge, S. (2011). Mnemonic control relies on a frontal-parietal-hippocampal network that is strengthened over childhood.

Paz-Alonso, P. M., Ghetti, S., Matlen, B. J., Anderson, M. C., \& Bunge, S. A. (2009). Memory suppression is an active process that improves over childhood. Frontiers in Human Neuroscience, 3, 24. doi:10.3389/neuro.09.024.2009.

Quay, H. C. (1997). Inhibition and attention deficit hyperactivity disorder. Journal of Abnormal Child Psychology, 25(1), 7-13.

Racsmány, M., Conway, M. A., \& Demeter, G. (2010). Consolidation of episodic memories during sleep: Long-term effects of retrieval practice. Psychological Science, 21(1), 80-85. doi:10.1177/0956797609354074.

Ramautar, J., Kok, A., \& Ridderinkhof, K. (2004). Effects of stop-signal probability in the stopsignal paradigm: The N2/P3 complex further validated. Brain and Cognition, 56(2), 234-252. doi:10.1016/j.bandc.2004.07.002.

Rescorla, R. A. (2004). Spontaneous recovery. Learning \& Memory, 11(5), 501-509. doi:10.1101/ $\operatorname{lm} .77504$.

Rubia, K., Overmeyer, S., Taylor, E., Brammer, M., Williams, S., Simmons, A., et al. (1999). Hypofrontality in attention deficit hyperactivity disorder during higher-order motor control: A study with functional MRI. American Journal of Psychiatry, 156, 891-896.

Rubia, K., Smith, A. B., Brammer, M. J., Tonne, B., \& Taylor, E. (2005). Abnormal brain activation during inhibition and error detection in medication-naive adolescents with ADHD. American Journal of Psychiatry, 162, 1067-1075.

Sahakyan, L., \& Kelley, C. (2002). A contextual change account of the directed forgetting effect. Journal of Experimental Psychology: Learning, Memory, and Cognition, 28(6), 1064-1072. doi:10.1037//0278-7393.28.6.1064.

Sahakyan, L., Waldum, E. R., Benjamin, A. S., \& Bickett, S. P. (2009). Where is the forgetting with list-method directed forgetting in recognition? Memory \& Cognition, 37(4), 464-476. doi:10.3758/MC.37.4.464.

Salamé, P., \& Danion, J. (2007). Inhibition of inappropriate responses is preserved in the ThinkNo-Think and impaired in the random number generation tasks in schizophrenia. Journal of the International Neuropsychological Society, 13(2), 277-287. doi:10.1017/S1355617707070300.

Sasaki, K., Gemba, H., \& Tsujimoto, T. (1989). Suppression of visually initiated hand movement by stimulation of the prefrontal cortex in the monkey. Brain Research, 495(1), 100-107.

Saunders, J., \& MacLeod, M. D. (2002). New evidence on the suggestibility of memory: The role of retrieval-induced forgetting in misinformation effects. Journal of Experimental Psychology: Applied, 8(2), 127-142.

Schmajuk, M., Liotti, M., Busse, L., \& Woldorff, M. G. (2006). Electrophysiological activity underlying inhibitory control processes in normal adults. Neuropsychologia, 44(3), 384-395. doi:10.1016/j.neuropsychologia.2005.06.005.

Schooler, J. W., Bendiksen, M., \& Ambadar, Z. (1997). Taking the middle line: Can we accommodate both fabricated and recovered memories of sexual abuse? In M. Conway (Ed.), Recovered Memories and False Memories. Oxford: Oxford University Press.

Schultz, T. M., Passmore, J., \& Yodor, C. Y. (2003). Emotional closeness with perpetrators and amnesia for child sexual abuse. Journal of Child Sexual Abuse, 12, 67-88.

Shobe, K. K., \& Schooler, J. W. (2001). Discovering fact and fiction: Case-based analyses of authentic and fabricated memories of abuse. In G. M. Davies \& T. Dalgleish (Eds.), Recovered memories: Seeking the middle ground (pp. 95-151). Chichester: Wiley.

Smith, M. (1993). Neurophysiological manifestations of recollective experience during recognition memory judgments. Journal of Cognitive Neuroscience, 5(1), 1-13.

Smith, M., \& Guster, K. (1993). Decomposition of recognition memory event-related potentials yields target, repetition, and retrieval effects. Electroencephalography and Clinical Neurophysiology, 86(5), 335-343. 
Smith, S. M., \& Moynan, S. C. (2008). Forgetting and recovering the unforgettable. Psychological Science, 19(5), 462-468. doi:10.1111/j.1467-9280.2008.02110.x.

Smith, S. M., \& Vela, E. (1991). Incubated reminiscence effects. Memory \& Cognition, 19(2), $168-176$.

Squire, L. R. (1992). Memory and the hippocampus: A synthesis from findings with rats, monkeys, and humans. Psychological Review, 99(2), 195-231.

Storm, B. C. (2010). Retrieval-induced forgetting and the resolution of competition. In A. Benjamin (Ed.), Successful remembering and successful forgetting: A festschrift in honor of Robert A. Bjork (pp. 89-105). New York: Psychology Press.

Storm, B. C., Bjork, E. L., Bjork, R. A., \& Nestojko, J. F. (2006). Is retrieval success a necessary condition for retrieval-induced forgetting? Psychonomic Bulletin and Review, 13(6), 1023-1027.

Storm, B. C., \& White, H. A. (2010). ADHD and retrieval-induced forgetting: Evidence for a deficit in the inhibitory control of memory. Memory, 18(3), 265-271. doi:10.1080/09658210903547884.

Tamm, L., Menon, V., Ringel, J., \& Reiss, A. (2004). Event-related fMRI evidence of frontotemporal involvement in aberrant response inhibition and task switching in attention-deficit/hyperactivity disorder. Journal of the American Academy of Child and Adolescent Psychiatry, 43(11), 1430-1440. doi:10.1097/01.chi.0000140452.51205.8d.

Tandoh, K., \& Naka, M. (2007). Durability of retrieval-induced forgetting. Shinrigaku Kenkyu: The Japanese Journal of Psychology, 78(3), 310-315.

Tomlinson, T. D., Huber, D. E., Rieth, C. A., \& Davelaar, E. J. (2009). An interference account of cue-independent forgetting in the no-think paradigm. Proceedings of the National Academy of Sciences of the United States of America, 106(37), 15588-15593. doi:10.1073/pnas.0813370106.

Tramoni, E., Aubert-Khalfa, S., Guye, M., Ranjeva, J. P., Felician, O., \& Ceccaldi, M. (2009). Hypo-retrieval and hyper-suppression mechanisms in functional amnesia. Neuropsychologia, 47(3), 611-624.

Trott, C. T., Friedman, D., Ritter, W., Fabiani, M., \& Snodgrass, J. G. (1999). Episodic priming and memory for temporal source: event-related potentials reveal age-related differences in prefrontal functioning. Psychology and Aging, 14, 390-413.

Underwood, B. J. (1949). Proactive inhibition as a function of time and degree of prior learning. Journal Of Experimental Psychology, 39(1), 24-34.

van Boxtel, G. J., van der Molen, M. W., Jennings, J. R., \& Brunia, C. H. (2001). A psychophysiological analysis of inhibitory motor control in the stop-signal paradigm. Biological Psychology, 58(3), 229-262.

Waldhauser, G.T., Johansson, M., \& Lindgren, M. (submitted). The effects of intentional suppression on recognition memory.

Waldhauser, G. T., Johansson, M., Backstrom, M., \& Mecklinger, A. (2011). Trait anxiety, working memory capacity, and the effectiveness of memory suppression. Scandinavian Journal of Psychology, 52(1), 21-27. doi:10.1111/j.1467-9450.2010.00845.x.

Wegner, D. M. (1994). Ironic processes of mental control. Psychological Review, 101, 34-52.

Wegner, D. M., Schneider, D. J., Carter, S., \& White, T. (1987). Paradoxical effects of thought suppression. Journal of Personality and Social Psychology, 53, 5-13.

Wenzlaff, R. M., \& Wegner, D. M. (2000). Thought suppression. In S. T. Fiske (Ed.), Annual review of psychology (Vol. 51, pp. 51-91). Palo Alto, CA: Annual Reviews.

Wessel, I., Huntjens, R. J. C., \& Verwoerd, J. R. L. (2010). Cognitive control and suppression of memories of an emotional film. Journal of Behavior Therapy and Experimental Psychiatry, 41(2), 83-89. doi:10.1016/j.jbtep. 2009.10.005.

Wessel, I., Wetzels, S., Jelicic, M., \& Merckelbach, H. (2005). Dissociation and memory suppression: A comparison of high and low dissociative individuals' performance on the Think-No Think task. Personality and Individual Differences, 39(8), 1461-1470.

Wheeler, M. A. (1995). Improvement in recall over time without repeated testing: Spontaneous recovery revisited. Journal of Experimental Psychology: Learning, Memory, and Cognition, 21(1), 173-184. 
Wilding, E. L., Doyle, M. C., \& Rugg, M. D. (1995). Recognition memory with and without retrieval of context: An event-related potential study. Neuropsychologia, 33(6), 743-767.

Wilding, E., \& Rugg, M. (1996). An event-related potential study of recognition memory with and without retrieval of source. Brain, 119, 889-905.

Williams, L. M. (1994). Recall of childhood trauma: A propective study of women's memories of child sexual abuse. Journal of Consulting and Clinical Psychology, 62(6), 1177-81.

Wilson, S. P., \& Kipp, K. (1998). The development of efficient inhibition: Evidence from directedforgetting tasks. Developmental Review, 18(1), 86-123.

Wylie, G. R., Fox, J. J., \& Taylor, T. L. (2008). Forgetting as an active process: An fMRI investigation of item-method directed forgetting. Cerebral Cortex, 18, 670-682. 